UW/PT 00-01, NORDITA 2000/79HE

\title{
Electroweak Bubble Nucleation, Nonperturbatively
}

\author{
Guy D. Moore \\ Department of Physics, University of Washington, Seattle WA 98195-1560 USA
}

\author{
Kari Rummukainen \\ NORDITA, Blegdamsvej 17, DK-2100 Copenhagen Ø, Denmark
}

(June 2000)

\begin{abstract}
We present a lattice method to compute bubble nucleation rates at radiatively induced first order phase transitions, in high temperature, weakly coupled field theories, nonperturbatively. A generalization of Langer's approach, it makes no recourse to saddle point expansions and includes completely the dynamical prefactor. We test the technique by applying it to the electroweak phase transition in the minimal standard model, at an unphysically small Higgs mass which gives a reasonably strong phase transition $\left(\lambda / g^{2}=0.036\right.$, which corresponds to $m_{H} / m_{W}=0.54$ at tree level but does not correspond to a positive physical Higgs mass when radiative effects of the top quark are included), and compare the results to older perturbative and other estimates. While two loop perturbation theory slightly under-estimates the strength of the transition measured by the latent heat, it over-estimates the amount of supercooling by a factor of 2 .
\end{abstract}

\section{INTRODUCTION}

Electroweak baryogenesis provides one of the best motivated and most testable mechanisms for the origin of the cosmological baryon number abundance. However, several ingredients are missing before we can make quantitative predictions. One set of needed ingredients is particle physics inputs. For instance, it is difficult to say much about electroweak matter at temperatures $T \sim 100 \mathrm{GeV}$, where electroweak symmetry is "restored" may occur, when we still do not know the Higgs mass. It is also absolutely necessary to know what other light $(m \lesssim 150 \mathrm{GeV})$ scalars there are, what couplings they have with the

\footnotetext{
${ }^{1}$ There is no qualitative distinction between the "symmetric" and "broken" electroweak phases, which are in fact analytically connected, and the symmetry is never truly "broken" even in vacuum; but when the phase transition is reasonably strong their quantitative behavior is very different. We will use the "symmetric" and "broken" terminology because it is convenient and widespread.
} 
Higgs boson(s), and what $\mathrm{CP}$ violation is operative at electroweak energy and temperature scales. Answering these questions will require new experimental results, and we will not have more to say about that here.

However, even if we knew the complete electroweak theory we could not at this time make accurate predictions of what baryon number would be cosmologically produced, because we do not have a complete set of reliable computational tools for studying the electroweak phase transition and the physical processes responsible for baryogenesis. This is best illustrated by briefly reviewing what the scenario is, and which aspects we do or do not currently have good control of.

Assuming a standard thermal history for the early universe back tof $T \sim 100 \mathrm{GeV}$, electroweak baryogenesis appears to be possible only if there is a fairly strong first order electroweak phase transition [2,[2]. The electroweak phase transition, if there is one, is radiatively induced, and determining its order and strength is a difficult problem with a long history. In summary, perturbation theory proves to be of some limited use when the phase transition is strong, but a reliable calculation of the strength of the electroweak phase transition requires a nonperturbative lattice calculation. The equipment for performing an accurate lattice calculation now exists, using either a 3-dimensional effective theory [3 [ 8] or 4-dimensional SU(2) gauge + Higgs theory [9, 10], so this part of the problem is solved.

If the electroweak phase transition is first order, then the universe will remain in the "symmetric" phase even after it is no longer thermodynamically favored. How deeply it supercools is the topic of this paper and we will return to it momentarily. After sufficiently deep supercooling, critical bubbles of the broken phase form at a cosmologically relevant rate, expand, and coalesce, completing the phase transition. [t It is the expansion of these bubbles into the symmetric phase which is expected to generate the baryon number. Specifically, the moving phase interface (bubble wall) can inject a $\mathrm{CP}$ violating flux of particles into the symmetric phase [12], where baryon number violation is efficient [13, 14]. Recently, the efficiency of the baryon number violation has been pinned down fairly accurately [15 19. However, neither the expansion of bubbles into the symmetric phase, nor the generation and propagation of $\mathrm{CP}$ violating particle fluxes, can yet be calculated with much precision or confidence, though there has been recent progress on both problems [20 23].

The bubble nucleation rate enters the final baryon number asymmetry by determining the amount of supercooling which occurs. The more supercooling, the greater the free energy difference between the phases, and the faster phase interfaces propagate. This in turn would mean a larger injected $\mathrm{CP}$ violating flux (since the flux must vanish in equilibrium in a $\mathrm{CPT}$ respecting theory). However, it would also mean that the phase interface would more quickly catch back up with particles it injected into the symmetric phase, which could have the reverse effect. The detailed dependence on supercooling and bubble wall velocity may be complicated, see for instance [24,23. How deep the supercooling proceeds can also be

\footnotetext{
${ }^{2}$ See 11 for an interesting discussion of what could happen if this assumption were not true.

${ }^{3}$ It has been argued that the phase transition can also proceed by coalescence of "subcritical bubbles" with almost no supercooling [11]. We feel our technique and results, presented here, put this idea to rest for the phase transition strength we are interested in.
} 
important because the universe heats during the phase transition, as the latent heat of the symmetric phase is released. We find below that the supercooling is less than in perturbation theory, while the latent heat is sometimes more; so this effect is more important than one might have anticipated. For the parameters we will study, there is enough latent heat, and little enough supercooling, that the universe reheats to the equilibrium temperature $T_{\text {eq }}$ before all space has converted to the broken phase. (The remaining space would convert much more slowly, as the expansion of the universe continues to absorb heat from the plasma.)

We are interested in a regime where the bubble nucleation rate is extremely small. This is because the phase transition completes when the bubble nucleation rate is, very roughly, around one bubble per Hubble volume per Hubble time. But at $T \sim 100 \mathrm{GeV}$, a Hubble time is $t_{\text {hubble }} \sim H^{-1} \sim m_{\mathrm{pl}} / T^{2} \sim 10^{17} / T$; so the rate of bubble nucleations must be $\sim\left(10^{-17} T\right)^{4}=10^{-68} T^{4}=e^{-157} T^{4}$. A more careful calculation, accounting for how much time the phase transition takes to complete, shows that the nucleation rate must be about $e^{-106} T^{4}$. When the nucleation rate is so small, it is "almost" a thermodynamical quantity, set by the free energy of the "critical bubble." This gives us a hint at how to determine it on the lattice; we must determine the free energy of a critical bubble. However, deciding what precisely this means and how to go about doing it, and relating the result to the real time rate for a rare process, require some work. In this paper we present a quantitative approach to address this problem, and we carry out our program for the minimal standard model, at zero Weinberg angle and an unphysical Higgs mass. Clearly this case will not be of direct physical interest. However, it allows us to determine how well the technique works, and to compare the bubble nucleation rate to what we would get using one of several less rigorous methods, such as a perturbative calculation of the critical bubble free energy or a "thin wall" treatment from either perturbative or nonperturbative inputs. We find that the "thin wall" treatment gives a reasonable answer but is not extremely accurate, while the perturbative approach is quite bad unless Higgs field wave function corrections are included.

Dynamical processes in a first order phase transition have been studied before with lattice simulations in the 3-dimensional Ising model [25]. However, in these studies the parameters of the simulations were chosen so that the nucleation rate was relatively large, so that the nucleation timescale was, at most, only few orders of magnitude larger than the microscopic interaction timescale. Thus, the nucleation process could be observed simply by waiting for the nucleation from a metastable to a stable state to happen during a straightforward standard (real-time) simulation. This is also the case for the recent work of Borsanyi et. al. [26].

However, in this work we are interested in extremely strongly suppressed nucleation. Indeed, very slow nucleation rates are a quite common characteristic of the first order phase transitions in Nature: fundamentally, this is due to the fact that the external parameters which drive the transition (temperature, say) usually vary on several orders of magnitude longer timescales than the microscopic interaction scale. This physical situation is out of reach of any numerical real-time simulation method relying on spontaneous appearance of bubbles in the metastable phase. On the other hand, the method presented in this work can be applied to an almost arbitrarily slow nucleation. This method has also been used with the 3-dimensional cubic anisotropy model [27].

An outline of the paper is as follows. In Section [1], we present the approach and discuss the obstacles. The discussion does not rely in any way on the specifics of the electroweak 
nucleation problem, except that it can be considered as a problem in classical statistical mechanics (both in the thermodynamics and in the dynamics of the system). Subsections ПA and ПB, together with subsection IVB, are the most important parts of the paper to understand; we encourage the reader to concentrate on them. In Section III, we review why it is true that the physics of the electroweak phase transition can be considered, both thermodynamically and dynamically, as a classical statistical mechanics problem. This section is a review of previous literature, included mostly to make the paper self-contained. In Section $\mathbb{I V}$ we present the numerical tools we use and the details of the calculation. It ends with a presentation of our results. Section $\nabla$ presents a number of alternative, "more perturbative" and less numerically intensive ways to try to determine the bubble nucleation rate, most of which have previously been used in the literature. We systematically compare these approaches to the nonperturbatively determined result, to analyze the reliability of the other approaches. Of these, the most trustworthy are the thin wall approximation using nonperturbative inputs (surface tension and latent heat), and two loop perturbation theory including Higgs field wave function corrections. Each of these approaches makes errors of order 20\%; other approaches, including those most widely used in the literature, give results off by almost a factor of 2. Finally, Section VI presents our conclusions.

\section{STRATEGY TO DETERMINE NUCLEATION RATE}

The next section will show why the calculation of the bubble nucleation rate at the electroweak phase transition is a problem in classical statistical mechanics. In this section we will assume this to be the case, and discuss the strategy for solving this statistical mechanics problem. The basic idea is that nucleation from the metastable symmetric phase to the stable broken phase is limited by the rarity of "critical bubble" configurations which lie in between. A "critical bubble" is, roughly, a configuration which in the medium termf is about equally likely to evolve towards the broken and homogeneous symmetric phases. An outline of the strategy is:

1. choose a measurable which will distinguish which field configurations are near the metastable symmetric phase, which are near the broken phase, and which are near the critical bubble;

2. evaluate the probability to be in the (exponentially rare) critical bubble configurations;

3. determine how quickly a "critical bubble" evolves towards one of the (meta)stable phases;

\footnotetext{
${ }^{4}$ We will distinguish three time scales. The short time scale is the longest time scale of typical thermalization processes in either phase, $\sim 1 / g^{4} T$. The long time scale is the time scale for nucleation to occur, $\sim e^{100} / T$. By the medium term we mean on a time scale well separated from the short and long time scales. At many points in our discussion there will be ambiguities of order the ratio of two of these time scales; but for strongly exponentially suppressed nucleation problems such ambiguities are tiny.
} 
4. determine the "dynamical prefactor," which tells what fraction of imputed "critical bubbles" really represent midpoints on a trajectory carrying the metastable symmetric phase to the stable broken one.

This section elucidates what we mean by each of the above, and why the whole approach is possible. Our strategy is similar to Langer's classic method [28], except that the saddle point treatment of the "critical" configurations is replaced by a Monte Carlo calculation, and we take care to treat completely the microscopic dynamics during "barrier crossing." Essentially the same technique has already been applied to determine the broken phase "sphaleron rate" in [29, 30, but we will not assume the reader is familiar with those papers.

Everything we say in this section is generic to first order phase transitions of liquid-gas type, ie. where there is no breaking of a global symmetry but the phases can be distinguished by the value of the volume average of a single, scalar local measurable. (However, this method is also fully applicable to transitions exhibiting a real global symmetry breaking.) In our case the volume averaged observable will be the average of the (length) ${ }^{2}$ of the Higgs field, $\phi_{\mathrm{av}}^{2} \equiv(1 / V) \int d^{3} x 2 \Phi^{\dagger} \Phi$. If it makes the reader more comfortable she can think of water and gas below the critical temperature, with the density as a measurable distinguishing the phases and pressure as a control parameter, or of a ferromagnet below the Curie temperature, with the magnetization as a measurable and an applied external field as a control parameter, rather than the electroweak model with $\phi_{\mathrm{av}}^{2}$ as a measurable and temperature as a control parameter. The big difference from the liquid gas system is that we know how to simulate the microscopic physics accurately even away from the second order endpoint, so Monte Carlo techniques applied to a first principles microscopic description of the thermodynamics can give reliable results.

With suitable generalizations the method described here can be applied to almost any metastable state decay problem, provided that

1. both the thermodynamics and the real time evolution of the system are amenable to numerical analysis,

2. there is an observable which can unambiguously distinguish the phases and can resolve the potential barrier between the them, and

3. the potential barrier is large and the tunnelling rate is small.

The first two conditions are quite generic, and if the third condition is not satisfied (for example, in "quenching" type problems), then one can make straightforward real-time simulation of the decay without relying on the special methods described here.

\section{A. General picture of homogeneous nucleation after weak supercooling}

Fix the ratio of the scalar self-coupling and gauge couplings, $\lambda / g^{2}$, to a value where there is a first order electroweak phase transition (or fix $T$ to a value below the Curie temperature for a ferromagnet, or $T$ below the critical temperature for a liquid-gas system), and ask how the canonical ensemble is distributed close to the critical temperature. In particular, consider how the constrained free energy $F\left(\phi_{\mathrm{av}}^{2}\right)$ varies as a function of

$$
\phi_{\mathrm{av}}^{2} \equiv \frac{1}{V} \int d^{3} x\left(2 \Phi^{\dagger} \Phi(x)-\text { counterterm }\right),
$$




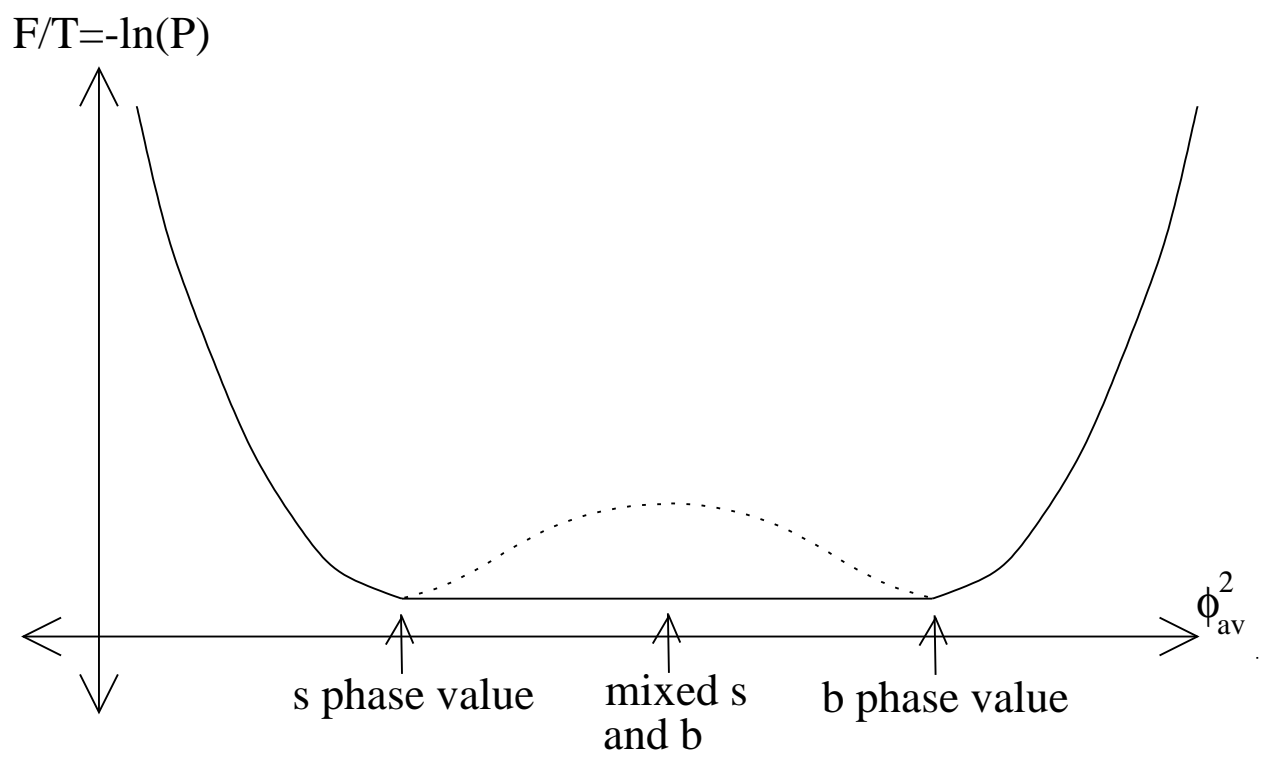

FIG. 1. Cartoon of how the constrained free energy $=-\log$ (probability of $\phi_{\mathrm{av}}^{2}$ ) varies with $\phi_{\mathrm{av}}^{2}$ at the equilibrium temperature in a large volume. The vertical axis gives minus the log of the fraction of states in the canonical ensemble with the given value of $\phi_{\mathrm{av}}^{2}$. The dotted line gives the free energy of a spatially homogeneous configuration with that value of $\phi_{\mathrm{av}}^{2}$; the truly most probable configurations at intermediate values are mixed phase configurations.

where the counterterm is needed to subtract UV divergences so the whole is well defined. We could consider any other measurable which has UV finite variance, but $\phi_{\mathrm{av}}^{2}$ will prove particularly convenient below. In a very large volume, at $T_{\text {eq }}$ (or zero external magnetic field in a ferromagnet, or the boiling pressure in a liquid-gas system), the constrained free energy density dependence on $\phi_{\mathrm{av}}^{2}$ will qualitatively resemble that of Fig. 1. The constrained free energy means $-T$ times the log of the weight of configurations in the canonical ensemble which have the specific value of $\phi_{\mathrm{av}}^{2}$. Very low or very high values of $\phi_{\mathrm{av}}^{2}$ are extremely rare; but values intermediate between the two bulk phases have free energy per volume equal to those of the bulk phases. This is because, besides the "expensive way" of getting an intermediate value of $\phi_{\mathrm{av}}^{2}$ — having $\Phi^{\dagger} \Phi$ equal the desired value homogeneously through the volume of interest - there is a "cheaper way," which is to have part of the volume be in one phase and the rest in the other phase. Then the disfavored intermediate values of $\Phi^{\dagger} \Phi$ are only achieved in an interface between the regions, whose volume does not scale extensively with the system volume. (Note that the fact that intermediate values of $\Phi^{\dagger} \Phi$ are disfavored, is exactly the statement that there is a first order phase transition with different values of $\Phi^{\dagger} \Phi$ in the two phases.)

What if we ask about a smaller volume, where the amount of space in the interface

\footnotetext{
${ }^{5}$ Strictly speaking, when we talk about spatial variations of $\Phi^{\dagger} \Phi$ or spatially homogeneous $\Phi^{\dagger} \Phi$ we actually mean a "coarse-grained" quantity: normally $\Phi^{\dagger} \Phi$ fluctuates wildly from point to point even in the pure symmetric or broken phases (it is, indeed, UV divergent). The coarse-grained $\Phi^{\dagger} \Phi$ is obtained by averaging over length scales $\approx$ bulk correlation length $\xi$. This is equivalent to
} 


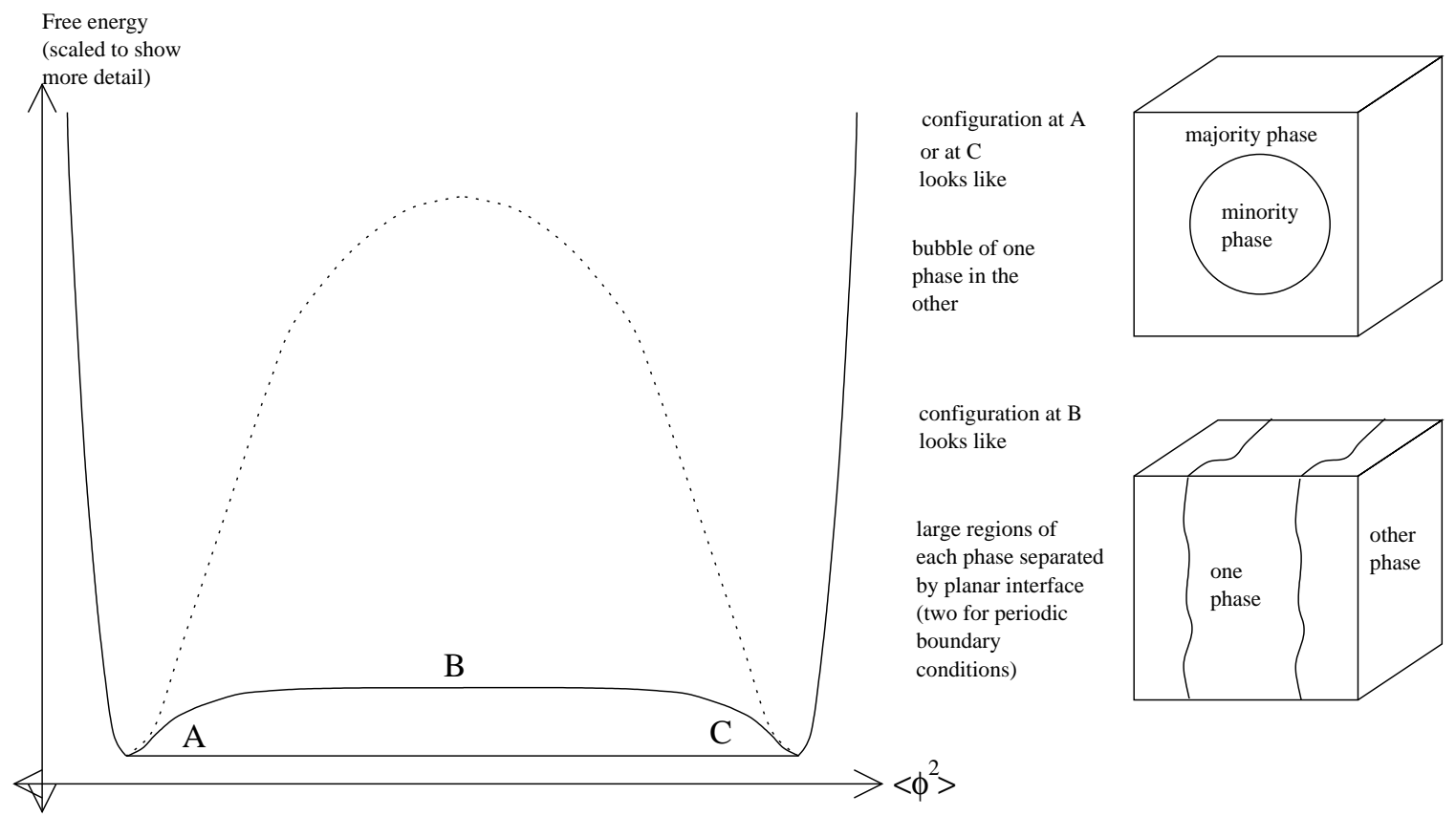

FIG. 2. Same as Fig. 1, but in a finite volume where the free energy of the interface between phases is not considered negligible. The free energy of a mixed phase state is higher than either pure phase because of the surface tension of the phase boundary. The figure also illustrates the physical appearances of the states which dominate the ensemble at intermediate values of $\phi_{\mathrm{av}}^{2}$.

between extensive phases is not negligible? A qualitative cartoon of the answer is given in Fig. 2. There is a free energy "barrier" between the two phases due to the free energy cost of the interface separating the two phases; but it is much lower than it would be if we had to stick with spatially homogeneous intermediate states. The barrier is roughly the surface tension of the interface times its area, which scales as the length squared of the box, while an extensive quantity would scale as length cubed.

The free energy at values of $\phi_{\mathrm{av}}^{2}$ between the stable phases gives us information about the free energy cost of mixed phase configurations; in particular, the free energy near the symmetric phase tells about the cost to have a small bubble of the broken phase in the symmetric phase. To see the value of this, in determining the rate of bubble nucleation, we now discuss how the picture changes when we change the temperature. The short answer is that one should "tip" Fig. 2, adding a linear in $\phi_{\text {av }}^{2}$ term to the free energy. In fact, for a special measurable this statement is exact, as we now discuss in some detail.

In the 3-D effective theory approximation we are working in (see next section), a variation $\delta T$ of the temperature corresponds to a change $\delta m_{H T}^{2}$ in the thermal Higgs mass squared. The size of the change can be read off from Eq. (3.3). Henceforth we will only talk about changing $m_{H T}^{2}$. The way that one determines the constrained free energy plots we have been discussing is that one finds the probability that a configuration drawn from the canonical ensemble has the value of $\phi_{\mathrm{av}}^{2}$ of interest. If we are interested in the free energy as a function

integrating out spatial momenta larger than $\xi^{-1}$. In the pure bulk phases the coarse-grained $\Phi^{\dagger} \Phi$ is almost homogeneous by construction, and non-homogeneous only in the mixed phase. 
of an operator $\mathcal{O}$, we want to know

$$
\frac{F\left(\mathcal{O}_{0}\right)}{T}=-\ln \int \mathcal{D}\left(A_{i}, \Phi\right) \exp (-H / T) \delta\left(\mathcal{O}(A, \Phi)-\mathcal{O}_{0}\right)
$$

up to an overall constant which is uninteresting (and depends on how we define the normalization of the path integral). For the special case that our operator is $\phi_{\mathrm{av}}^{2}$, the constrained free energy has an extremely convenient property. Observe from Eq. (3.7) that the way that $m_{H T}^{2}$ enters the Hamiltonian is

$$
\begin{aligned}
H & =H_{m=m_{0}}+\frac{m_{H T}^{2}-m_{0}^{2}}{2} \int d^{3} x 2 \Phi^{\dagger} \Phi(x), \\
& =H_{m=m_{0}}+\frac{m_{H T}^{2}-m_{0}^{2}}{2} V \phi_{\mathrm{av}}^{2},
\end{aligned}
$$

with $m_{0}^{2}$ any particular value we might choose. The constrained free energy as a function of $\phi_{\mathrm{av}}^{2}$ is

$$
\frac{F\left(\phi_{\mathrm{av}, 0}^{2}\right)}{T}=-\ln \int \mathcal{D}\left(A_{i}, \Phi\right) e^{-H_{m=m_{0}} / T} e^{-\left(m_{H T}^{2}-m_{0}^{2}\right) V \phi_{\mathrm{av}}^{2} / 2 T} \delta\left(\phi_{\mathrm{av}}^{2}-\phi_{\mathrm{av}, 0}^{2}\right)
$$

but we can now use the delta function to replace $\phi_{\mathrm{av}}^{2}$ in the exponential with $\phi_{\mathrm{av}, 0}^{2}$, which is not integrated over; pulling it out of the integral gives

$$
\begin{aligned}
\frac{F\left(\phi_{\mathrm{av}, 0}^{2}\right)}{T} & =-\ln e^{-\left(m_{H T}^{2}-m_{0}^{2}\right) V \phi_{\mathrm{av}, 0}^{2} / 2 T} \int \mathcal{D}\left(A_{i}, \Phi\right) e^{-H_{m=m_{0}} / T} \delta\left(\phi_{\mathrm{av}}^{2}-\phi_{\mathrm{av}, 0}^{2}\right) \\
& =\left[\frac{\left(m_{H T}^{2}-m_{0}^{2}\right) V}{2 T} \phi_{\mathrm{av}, 0}^{2}\right]-\ln \int \mathcal{D}\left(A_{i}, \Phi\right) e^{-H_{m=m_{0}} / T} \delta\left(\phi_{\mathrm{av}}^{2}-\phi_{\mathrm{av}, 0}^{2}\right) .
\end{aligned}
$$

The second term here is independent of $m_{H T}^{2}$; it can be determined once and used at any value of $m_{H T}^{2}$ thereafter. Hence the effect on $F\left(\phi_{\mathrm{av}}^{2}\right)$ of shifting $m_{H T}^{2}$ is very simple; it just adds an extensive, linear in $\phi_{\text {av }}^{2}$ term to $F$. (The same thing would happen if we considered the magnetization in a ferromagnetic system where we vary the external magnetic field, or the density in a liquid-gas system where we vary the pressure. The key is to consider a measurable which appears in the Hamiltonian next to the control parameter which takes us through the transition.) If we used a different measurable the qualitative behavior would be the same-the free energy as a function of that measurable would be roughly a "tilted" version of its $T_{\text {eq }}$ appearance-but this would not hold as an exact quantitative statement.

Now consider how the free energy plot looks when we shift $m_{H T}^{2}$. A cartoon is provided in Fig. 3. For small amounts of supercooling, the symmetric phase minimum shifts over slightly, but persists as a local minimum of the free energy. It is labeled $(A)$ in the figure. Since we will be concerned with classical dynamics, for an element of the thermal ensemble at $(A)$ to get to the global minimum, it must pass through $(B),(C)$, and $(D)$. The time evolution of a configuration at $(B)$ is almost certain, in the medium term, not to go to $(C)$, because $F / T$ is $-\log$ (Probability); there are vastly more states with $\phi_{\text {av }}^{2}$ equal the value at $(B)$ than the value at $(C)$, so only a tiny fraction will evolve to $(C)$ in the medium term, since time evolution preserves the canonical ensemble. On the other hand, configurations at $(D)$ are almost certain not to "go back," and will continue to the broken phase minimum. 


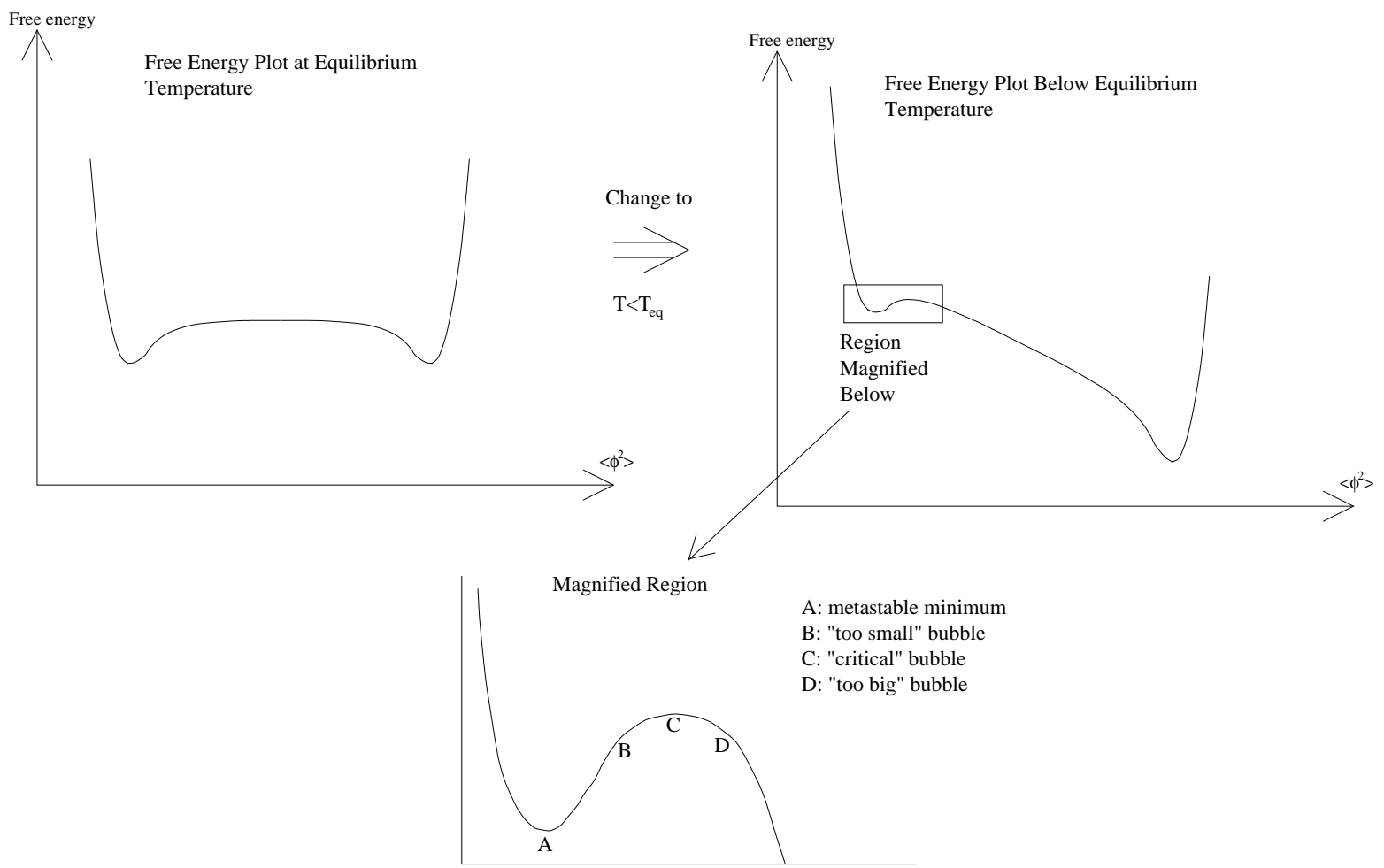

FIG. 3. Cartoon showing how free energy in a finite box changes when we lower the temperature. For small temperature changes, both minima survive, but one (A) is no longer globally stable. The least likely configuration on the way to the stable minimum is $(\mathrm{C})$ the critical bubble: its unlikelihood restrains the rate at which configurations near (A) go to the true minimum.

It is the rarity of configurations at $(C)$ which limits the rate at which configurations near $(A)$ time evolve into the broken phase. If it were true that every configuration at $(C)$ were on the way from $(A)$ to the broken phase (or going from the broken phase to $(A)$ ), then with a little dynamical information we could determine the nucleation rate. It may be, though, that most configurations at $(C)$ are either coming from $(B)$ and going back there, or coming from $(D)$ and going back there. (This might happen if the choice of measurable is not optimal, for instance.) In this case, nucleations from the symmetric to the broken phase would be even rarer than the free energy of states at $(C)$ implies. So the rarity of configurations at $(C)$ provides an upper bound on the nucleation rate, which can be turned into a determination with some additional dynamical information.

\section{B. Real time rate, dynamical prefactor}

Now we discuss how to turn the discussion and cartoons of the last section into a calculation of a real time rate for nucleations. Suppose we have, by multicanonical tools discussed in subsection $\mathbb{E B}$, computed the constrained free energy as a function of some measurable, which we take to be $\phi_{\mathrm{av}}^{2}$. It is also possible for us to collect a sample of the canonical ensemble restricted to some narrow range of $\phi_{\mathrm{av}}^{2}$, for instance, the range right around $\phi_{\mathrm{av}, \mathrm{C}}^{2}$, the least likely value of $\phi_{\mathrm{av}}^{2}$. What to we do with them? 
The first thing to do is to determine how exponentially suppressed critical bubbles are. But the answer depends on the measurable and does not give a real time rate. The second step is to determine what the flux of states in the canonical ensemble through the critical bubble is, normalized to the probability to be in the symmetric phase. That is, we should determine

$$
\text { probability flux } \equiv \frac{\mathrm{P}\left(\left|\phi_{\mathrm{av}}^{2}-\phi_{\mathrm{av}, \mathrm{C}}^{2}\right|<\epsilon / 2\right)}{\epsilon \mathrm{P}\left(\phi_{\mathrm{av}}^{2}<\phi_{\mathrm{av}, \mathrm{C}}^{2}\right)} \times\left\langle\left|\frac{\Delta \phi_{\mathrm{av}}^{2}}{\Delta t}\right|_{\phi_{\mathrm{av}, \mathrm{C}}^{2}}\right\rangle,
$$

with $\mathrm{P}$ (condition) denoting the fraction of the canonical ensemble which satisfies the condition, and with $\epsilon$ infinitesimal. The first term here is the probability density to be at the critical value $\phi_{\mathrm{av}, \mathrm{C}}^{2}$ of $\phi_{\mathrm{av}}^{2}$, which is our "definition" of the critical bubble. It could equally be written as

$$
\frac{\mathrm{P}\left(\left|\phi_{\mathrm{av}}^{2}-\phi_{\mathrm{av}, \mathrm{C}}^{2}\right|<\epsilon / 2\right)}{\epsilon \mathrm{P}\left(\phi_{\mathrm{av}}^{2}<\phi_{\mathrm{av}, \mathrm{C}}^{2}\right)}=\left[\int_{\mathrm{small}}^{\phi_{\mathrm{av}, \mathrm{C}}^{2}} \exp \left(\frac{F\left(\phi_{\mathrm{av}, \mathrm{C}}^{2}\right)-F\left(\phi_{\mathrm{av}}^{2}\right)}{T}\right) d \phi_{\mathrm{av}}^{2}\right]^{-1},
$$

and will be evaluated by Monte Carlo. The second term in Eq. (2.6) is the mean change, in absolute magnitude, of $\phi_{\mathrm{av}}^{2}$ in a time interval $\Delta t$, sampled over configurations at the critical bubble. Provided we take $\Delta t$ shorter than any typical infrared scale - in particular it should be shorter than the time scale to go from a configuration with $\phi_{\mathrm{av}}^{2}=\phi_{\mathrm{av}, \mathrm{C}}^{2}$ to one where $F / T$ differs by order 1 - then the ratio will tell the number of configurations which pass from one side of the critical bubble to the other in a time interval $\Delta t$, times the time interval and divided by the number of symmetric phase configurations. In other words, the combination gives the flux of configurations in the canonical ensemble through the critical bubble.

This flux is NOT the bubble nucleation rate we are after, though it is clearly an upper bound. We have to multiply by the fraction of critical bubble crossings which actually mediate a change from phase to phase. To this end we define a "dynamical prefactor," $\mathbf{d}$, as

$$
\mathbf{d} \equiv \frac{\text { trajectories getting from }(B) \text { to }(D)}{\text { crossings of }(C)} .
$$

In other words, if we consider all real time trajectories, $\mathbf{d}$ is the fraction of crossings of $(C)$ which represent "permanent changes" from one side to the other of the barrier. To determine it we sample the ensemble of configurations restricted to those at the critical bubble, and for each element of this ensemble we construct a trajectory forward and backwards in time, long enough to see the configuration come from and go to an exponentially more common value of $\phi_{\mathrm{av}}^{2}$. Sampling trajectories every $\Delta t, \mathbf{d}$ is

$$
\mathbf{d}=\left\langle\frac{1}{\# \text { of crossings }} \times\left\{\begin{array}{ll}
1 & \text { change sides } \\
0 & \text { don't }
\end{array}\right\rangle\right.
$$

The average is over the canonical ensemble, restricted to configurations with $\left|\phi_{\mathrm{av}}^{2}-\phi_{\mathrm{av}, \mathrm{C}}^{2}\right|<$ $\epsilon / 2$, and over trajectories through those configurations. The measure to be used is the canonical one, times $\left|\Delta \phi_{\mathrm{av}}^{2} / \Delta t\right|$ evaluated where the crossing takes place; so the sample is 
precisely the sample of the flux of states through $\phi_{\mathrm{av}}^{2}=\phi_{\mathrm{av}, \mathrm{C}}^{2}$. In Eq. (2.9), (\# of crossings) means the number of crossings of the critical bubble a trajectory makes in the medium term. To determine this we need to follow the trajectory until it reaches an exponentially more common value of $\phi_{\mathrm{av}}^{2}$, ie. point $(B)$ or $(D)$ in Fig. 3. 3 . The trajectory "changes sides" if it goes from $(B)$ to $(D)$ or vice versa, and does not change sides if it returns to the same side it came from. In our case, the real time evolution will be Langevin evolution, see next section, and the forward and backwards time evolutions are just two Langevin evolutions with two different realizations of the random force. For the case of Hamiltonian dynamics, the forward evolution would be evolution with a set of momenta drawn from the canonical ensemble and the backwards evolution would be evolution with the same momenta, but sign reversed ( $\mathrm{T}$ conjugated). In either case we are approximating the expectation values in Eq. (2.9) with an average over a sample of trajectories, ie. we take the average in Eq. (2.9) by a Monte Carlo integration. We discuss why this procedure gives the correct nucleation rate at more length in Appendix A.

For Hamiltonian dynamics, in an UV regulated theory, both $\mathbf{d}$ and $\left\langle\left|\Delta \phi_{\mathrm{av}}^{2} / \Delta t\right|\right\rangle$ should have well defined small $\Delta t$ limits. This is not the case for Langevin dynamics, however. If we sample a Langevin trajectory with a smaller $\Delta t$, the number of crossing should grow, as each crossing gets "resolved" into potentially more; this is a normal feature of a Brownian path. However, $\left\langle\left|\Delta \phi_{\mathrm{av}}^{2} / \Delta t\right|\right\rangle$ will also depend on $\Delta t$ by a compensating amount. For suitably short $\Delta t$, the time history of $\phi_{\mathrm{av}}^{2}$ near each crossing looks like a Brownian random walk. By well known properties of Brownian random walks, $\left\langle\left|\Delta \phi_{\mathrm{av}}^{2} / \Delta t\right|\right\rangle$ scales as $(\Delta t)^{-1 / 2}$, while $\mathbf{d}$ scales as $(\Delta t)^{1 / 2}$, and the product has a finite small $\Delta t$ limit. Hence, for Langevin dynamics, neither $\mathbf{d}$ nor $\left\langle\left|\Delta \phi_{\mathrm{av}}^{2} / \Delta t\right|\right\rangle$ are well defined but the product is.

It is finally the product,

$$
\frac{\text { nucleation rate }}{\text { Volume }}=\frac{1}{2 V} \text { probability flux } \times \mathbf{d},
$$

which we are interested in. The factor of $(1 / 2)$ is because half of the permanent crossings the algorithm finds are into the symmetric phase. The factor $1 / V$ turns the nucleation rate into a rate per unit volume.

\section{Complications: peculiar behavior in finite volumes}

In this subsection we discuss some complications with applying our technique, arising from finite volume effects. The conclusion will be that the volume must be fairly large, so the bubble interior fills at most about $15 \%$ of it; and that as a consequence, it is best to choose a measurable with a very small variance in the metastable phase. The impatient reader may want to skip this section and just accept that conclusion.

\footnotetext{
${ }^{6}$ We must also check that this criterion is sufficient to ensure that it is very unlikely for the trajectory to return again to $(C)$, which is not necessarily ensured; the ensemble of configurations at $(B)$ which have just evolved from configurations at $(C)$ is not the same as the ensemble of all configurations at $(B)$. In practice this does not prove to be a problem.
} 
What we want is the nucleation rate in very large volumes. In practice it is impossible to work directly in a very large volume, for reasons of numerical cost. Naively the cost of performing the Monte Carlo calculation scales as the volume, but in practice the scaling is still more severe, because of memory and communication costs and because the Monte Carlo becomes less efficient in large volumes, particularly at the value of $\phi_{\mathrm{av}}^{2}$ where the typical configuration changes from being homogeneous, supercooled symmetric phase to being an isolated bubble.

a. Resolving the critical bubble: There is another reason why we would like to work in a small volume. This is because any volume averaged measurable, for example $\phi_{\mathrm{av}}^{2}$, fluctuates also in the pure symmetric or broken phase. The width of these fluctuations behave as $1 / \sqrt{V}, V$ the volume, whereas the contribution of a fixed size bubble to $\phi_{\mathrm{av}}^{2}$ scales as $1 / V$. Thus, if we keep the size of the critical bubble constant but increase $V$, the fluctuations of $\phi_{\mathrm{av}}^{2}$ in the pure symmetric phase outside of the bubble increase and degrade the the "cleanliness" of $\phi_{\mathrm{av}}^{2}$ as a description of the critical bubble.

Concretely, what we mean by this degradation is that the more symmetric phase there is, the more likely it is that the value of $\phi_{\mathrm{av}}^{2}$ consistent with the critical bubble (point $\mathrm{C}$ in Fig. (3) really arises from too small a bubble plus an upward fluctuation in the symmetric phase contribution to $\phi_{\mathrm{av}}^{2}$, or too big a bubble and a downward fluctuation. The result is that, as we increase the volume, the measured free energy of the bubble should fall somewhat faster than the "true" bubble free energy (which decreases as $-\log (V)$, due to the translational zero modes of the bubble configuration). However, the measured value of the "dynamical prefactor" d, Eq. (2.9), should become smaller, since it is more likely that an imputed critical bubble is really on one or the other side and will begin and end at the same phase. These effects cancel exactly, so that the nucleation rate has a well-defined infinite volume limit. However, as $\mathbf{d}$ becomes smaller, it takes more work to measure it with good relative accuracy. This effect also degrades the efficiency of the Monte Carlo.

b. Maximum size of the critical bubble: For the above reasons it is to our advantage to use as small a volume for a given size bubble as we can get away with. Naively, this means we should use the smallest volume for which the critical bubble is unable to "see itself" around our periodic boundary conditions (we choose to work in a cubic box with periodic boundary conditions. It appears necessary to use a box which has everywhere a flat spatial metric, and does not have boundaries, because either effect could modify the bubble free energy.). Very naively, this means we must ensure that the radius of the critical bubble $r_{\max }$ is less than half the box length $L, r_{\max }<L / 2$.

In fact the criterion for a sufficiently large $L$ is more severe than $L>2 r_{\max }$. The reason is that a spherical broken phase bubble with $L / 3<r<L / 2$ is at best only metastable: a configuration with a cylinder of the broken phase, extending through the length of the box and having the same volume as the bubble, will have smaller phase interface area and hence smaller total free energy. To compare the favorability of different geometries as a function of broken phase volume fraction, we will make a "thin wall" approximation in which the interface between phases is treated as a geometrical surface, and the free energy is equal to its area times the surface tension: $F=\sigma A$. This approximation is correct in the limit that the box size $L$ is much larger than the wall thickness. Though our simulations will not be strictly in this limit, it is a suitable approximation for understanding the relative favorabilities of different mixed phase geometries. One can then write down how the area 


\begin{tabular}{|c|c|c|c|}
\hline Geometry & Sphere & Cylinder & Planes \\
\hline Area $(r)$ & $4 \pi r^{2}$ & $2 \pi r L$ & $2 L^{2}$ \\
\hline Volume $(r)$ & $\frac{4 \pi}{3} r^{3}$ & $\pi r^{2} L$ & any \\
\hline$A(V)$ & $\left(36 \pi V^{2}\right)^{1 / 3}$ & $2 \sqrt{\pi L V}$ & $2 L^{2}$ \\
\hline Volumes where stable & $0-4 \pi / 81$ & $4 \pi / 81-1 / \pi$ & $1 / \pi-(1-1 / \pi)$ \\
\hline Vol. where metastable & $0-\pi / 6$ & $1 / 4 \pi-\pi / 4$ & $0-1$ \\
\hline
\end{tabular}

TABLE I. Area and volume as functions of $r$, and area as function of volume, for each possible geometry for phase coexistence; and derived volume range where the geometry is preferred and where it is metastable in the strict thin wall limit. The upper end of the sphere and cylinder metastability ranges are where they touch themselves across the periodic boundary conditions. The lower metastability limit on the cylinder is where it becomes unstable to a $\delta r=\sin (2 \pi z / L)$ excitation.

and volume vary as a function of radius for a sphere and a cylinder extending the length of

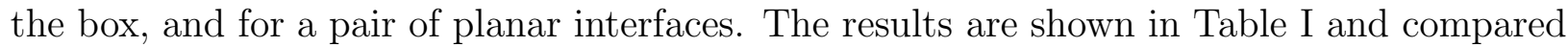
in Figure 1 . Equating the areas at fixed volume,

$$
A(V)_{\text {sphere }}=\left(36 \pi V^{2}\right)^{1 / 3}=\sqrt{4 \pi L V}=A(V)_{\text {cylinder }},
$$

we find that the sphere and cylinder are degenerate for $V=L^{3} \times 4 \pi / 81 \simeq 0.155 L^{3}$, and the cylinder and planes are degenerate for $V=L^{3} / \pi$. A plot of the free energy in a very large box should approximately resemble the solid line in Fig. 4 . It will have slope discontinuities where one geometry gives way to the next. The metastability of one geometry against another can be quite strong, and this makes it challenging to perform a multicanonical Monte Carlo determination of the free energy in a volume large enough that the interfaces are thin compared to $L$, unless one only wants the plot in the region where one geometry is relevant. (Fortunately this will be the case for us.)

Since both the cylinder and the planar geometry have free energies which clearly depend essentially on the box geometry and volume, the part of the free energy plot where they dominate describes finite volume artifacts rather than physics which has any correspondence to that at larger volumes. However, the radius of the sphere where the cylinder becomes equally favorable is only $r=L / 3$, safely small enough that the sphere will not "see itself" around the periodic boundary conditions (unless $L$ is only a few times the interface width). Hence we can expect the sphere geometry, until it stops being the favored geometry, not to care about the periodic boundary conditions but to represent more or less faithfully the behavior of an isolated bubble in a larger volume.

We may also expect that, in a suitably large volume, the transition rate between the spherical and cylindrical geometries is sufficiently slow that we could rely on the metastability of the sphere to explore larger radii than $r=L / 3$. However we will conservatively not do so in what follows, but will restrict ourselves to such volume and bubble size combinations that the critical bubble we obtain will have $\phi_{\mathrm{av}}^{2}<0.15 \phi_{\mathrm{av}}^{2}$ (broken) $+0.85 \phi_{\mathrm{av}}^{2}$ (symmetric). We will also check to see that the bubbles we analyze are approximately spherical and not cylindrical and that they do not touch across the periodic boundary. 


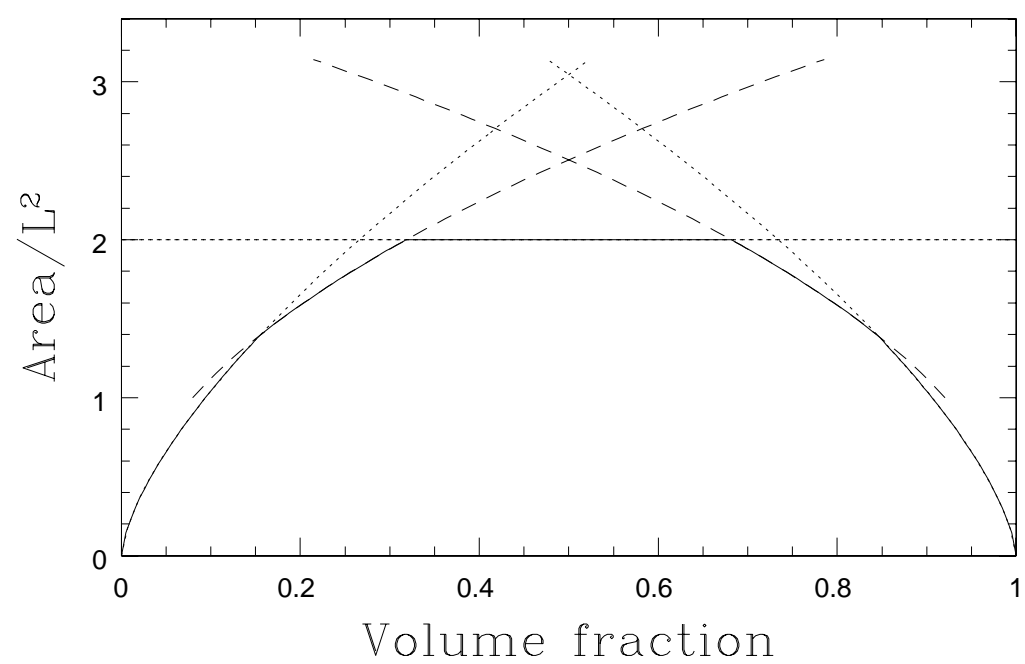

FIG. 4. Area, and hence free energy, as a function of volume fraction, in the thin wall approximation. The solid line is the minimum over interface geometries; the large volume free energy curve would follow the solid line. Dotted lines are the metastable extensions of the sphere geometry (sloping) or the planar boundary geometry (flat), while the dashed lines show metastable extensions of the cylindrical geometry.

\section{ELECTROWEAK BUBBLE NUCLEATION AS A PROBLEM IN CLASSICAL STATISTICAL MECHANICS}

In this section we briefly review why we can view the bubble nucleation problem as a problem in classical statistical mechanics for Yang-Mills Higgs field theory. Nothing in this section is new; it reviews the last few years' developments both in the thermodynamics of the electroweak phase transition and in the dynamics of infrared Yang-Mills Higgs fields. We include it here to make the paper more self-contained. Readers who are already familiar with this material may want to skip this section.

\section{A. Thermodynamics: dimensional reduction}

Here we review how the thermodynamics of infrared fields in the $\mathrm{SU}(2)$ sector of the standard model is well approximated by a 3 dimensional path integral, which is the same as the partition function of classical $3+1$ dimensional SU(2) Higgs theory at finite temperature (and with suitable regulation and counterterms).

The full theory we are interested in has thermodynamics described by the path integral (Lorentz indices are Euclidean with positive metric; Greek indices range over space and time, Latin indices $i, j, k$ only over space, indices $a, b, c$ are $\mathrm{SU}(2)$ group indices) 


$$
\begin{aligned}
Z=\int \mathcal{D}\left(\Phi, A_{\mu}, \text { etc }\right) & \exp \left(-S_{\mathrm{E}} / \hbar\right), \\
S_{\mathrm{E}}=\int_{0}^{\hbar / T} d \tau \int d^{3} x[ & \frac{1}{4 g^{2}} F_{\mu \nu}^{a} F_{a}^{\mu \nu}+\left(D_{\mu} \Phi\right)^{\dagger}\left(D^{\mu} \Phi\right)+\left(m_{H o}^{2}+\lambda \Phi^{\dagger} \Phi\right) \Phi^{\dagger} \Phi \\
& + \text { hypercharge }+ \text { fermions }+ \text { glue }] .
\end{aligned}
$$

Here and throughout $g$ is $g_{w}$ the weak coupling. The $\tau$ integration has periodic boundary conditions for bosons and antiperiodic boundary conditions for fermions. Beginning here we will neglect hypercharge, to simplify things. This is not too bad an approximation 31. Including it would be a straightforward extension of what we discuss.

There are two things to observe right away about this theory. First, mean field theory predicts that if $m_{H o}^{2}$ changes, there is a second order phase transition. Second, at the scale $T$ (which will be of order the weak scale, $T \sim 80 \mathrm{GeV}$ ), the coupling is weak. This is just the statement that the weak sector of the standard model is indeed weakly coupled. Hence, if there is a phase transition, barring some large hierarchy of couplings such as $\lambda / g^{2} \ll 1$, it will be weak, and correlation lengths will be $\xi \gg 1 / T$.

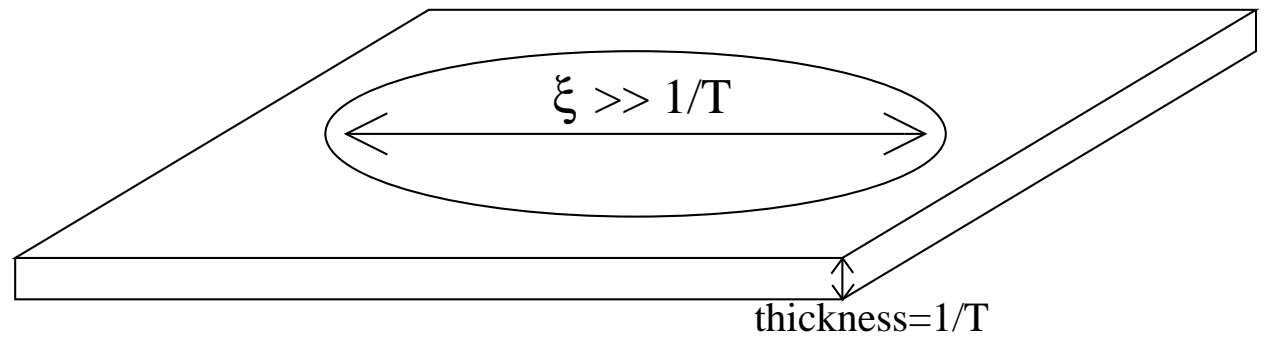

FIG. 5. Cartoon of how a $3+1$ dimensional spacetime, $\Re^{3} \times S^{1}$, drawn here as $2+1$ dimensional, can look effectively 3 dimensional for long distances.

As motivated in Fig. 河, at such infrared scales the effective behavior is 3 dimensional. This is just because any field varying only on the length scale $\xi \gg 1 / T$ will not vary appreciably across the Euclidean time width of the "slab." One sees this formally by Fourier transforming the $\tau$ direction in Eq. (3.1). The Euclidean frequencies arise from transforming a compact range and so are discrete: $\left(\partial_{\tau} \Phi(\tau)\right)^{2}$ becomes $(2 \pi n T)^{2} \Phi_{n}^{2}$ for bosons, while $\bar{\psi} \gamma^{0} \partial_{\tau} \psi(\tau)$ becomes $((2 n+1) \pi T) \bar{\psi}_{n} \gamma_{0} \psi_{n}$ for fermions. All but the $n=0$ bosonic mode are very heavy and can be integrated out. The result (continuing to use 4 dimensional notation for fields and couplings) is

$$
\begin{aligned}
\frac{S_{\mathrm{E}}}{\hbar}=\frac{1}{T} \int d^{3} x & {\left[\frac{1}{4 g^{2}} F_{i j}^{a} F_{a}^{i j}+\frac{1}{2}\left(D_{i} A_{0}\right)^{a}\left(D_{i} A_{0}\right)^{a}+\left(D_{i} \Phi\right)^{\dagger}\left(D_{i} \Phi\right)+m_{H T}^{2} \Phi^{\dagger} \Phi+\lambda\left(\Phi^{\dagger} \Phi\right)^{2}\right.} \\
& \left.+m_{\mathrm{D}}^{2} A_{0}^{a} A_{0}^{a}+\frac{\left(g^{2}+O\left(g^{2} \alpha_{w}\right)\right)}{4} A_{0}^{a} A_{0}^{a} \Phi^{\dagger} \Phi+O\left(g^{2} \alpha_{w}\right)\left(A_{0}^{a} A_{0}^{a}\right)^{2}+\operatorname{Dim} 6\right],
\end{aligned}
$$

where "Dim 6" indicates dimension 6 and higher induced operators, which have an irrelevantly small effect on the infrared physics, so we immediately drop them. The fields we write here correspond to the zero frequency components of the 4 dimensional fields, ie. $\Phi$ in 
Eq. (3.2) is $T \int d \tau \Phi(x, \tau)$, with $\Phi(x, \tau)$ the field appearing in the path integral in Eq. (3.1). In this expression $\lambda$ and $g^{2}$ are couplings of an effective $3 D$ theory, which (after dropping dimension 6 operators) is super-renormalizable; they do not run. Their relation to the coefficients of the full theory, and a detailed discussion of the matching procedure used to derive them, is given in [5]. In particular we mention that the Higgs mass squared, $m_{H T}^{2}$, receives $O\left(g^{2} T^{2}\right)$ positive thermal corrections;

$$
m_{H T}^{2}=m_{\mathrm{vac}, \mathrm{ren}}^{2}+\frac{3 g^{2}+4 y_{t}^{2}+8 \lambda}{16} T^{2}+\left(\mu \text { dependent } O\left(g^{4} T^{2}\right)\right) .
$$

Here $y_{t}$ is the top quark Yukawa coupling. Since $m_{\text {vac,ren }}$ is negative, varying $T$ can change the sign of the Higgs mass squared and induce a phase transition. For generic values of $\lambda / g^{2}$, correlation lengths at the phase transition are of order $\xi \sim 1 / g^{2} T$, which is why we can drop the dimension 6 operators.

Note that the form of $S_{\mathrm{E}} / \hbar$ looks very much like the Boltzmann factor, $E / T$, of a classical theory. The main difference is that in classical SU(2) Higgs theory, we expect the electric field strength and the Higgs field momentum $\Pi$, rather than $A_{0}$, to appear;

$$
\begin{aligned}
Z_{\mathrm{cl}} & =\int \mathcal{D}\left(E_{i}^{a}, A_{i}^{a}, \Phi, \Pi\right) \exp (-H / T) \delta\left(\left(D_{i} E_{i}\right)^{a}+\left[\Phi^{\dagger} \frac{i g \tau^{a}}{2} \Pi+\text { c.c. }\right]\right), \\
H & =\int d^{3} x\left[\frac{1}{4 g^{2}} F_{i j}^{a} F_{a}^{i j}+\frac{1}{2} E_{i}^{a} E_{i}^{a}+\Pi^{\dagger} \Pi+\left(D_{i} \Phi\right)^{\dagger}\left(D_{i} \Phi\right)+m_{H T}^{2} \Phi^{\dagger} \Phi+\lambda\left(\Phi^{\dagger} \Phi\right)^{2}\right] .
\end{aligned}
$$

Here the delta function enforces Gauss' Law. But as noted by Ambjørn and Krasnitz [32], if we implement Gauss' Law by introducing a Lagrange multiplier, which we suggestively name $A_{0}$,

$$
\delta(\ldots)=\int \mathcal{D} A_{0} \exp \left(i \int d^{3} x A_{0}^{a}\left(\left(D_{i} E_{i}\right)^{a}+\left[\Phi^{\dagger} \frac{i g \tau^{a}}{2} \Pi+\text { c.c. }\right]\right) / T\right),
$$

then the $E$ and $\Pi$ integrations are Gaussian and can be performed, generating

$$
H \supset \frac{1}{2}\left(D_{i} A_{0}\right)^{a}\left(D_{i} A_{0}\right)^{a}+\frac{g^{2}}{4} A_{0}^{a} A_{0}^{a} \Phi^{\dagger} \Phi+(0+\text { radiatively induced }) A_{0}^{a} A_{0}^{a} .
$$

Here the bare Debye mass is zero but one is radiatively induced, with a linear divergent coefficient in any regulation where such terms do not identically vanish (such as the lattice). Hence, the thermodynamics of the full theory DO look like those of the classical theory, except that the Debye mass of the classical theory is radiatively induced and regulation dependent, and there are very small $O\left(g^{2} \alpha_{w}\right)$ extra interaction terms involving the $A_{0}$ field, present in the actual thermodynamics but not in the thermodynamics of the classical system.

It is a good approximation, for the thermodynamics both of the classical theory and of the dimensionally reduced full theory, to integrate out the $A_{0}$ field, including it by the radiative

\footnotetext{
${ }^{7}$ Note that both $m_{H T}^{2}$ and $m_{\mathrm{D}}^{2} \sim g^{2} T^{2}$ renormalize logarithmically at the two loop level, $\left(\partial m_{H T}^{2} / \partial \ln \mu\right) \sim g^{4} T^{2}$, so the sign of $m_{H T}^{2}$ near the transition is actually renormalization point dependent.
} 
corrections it will induce in the remaining couplings. In this approximation one shifts slightly the coefficients of the terms in Eq. (3.2) which do not contain $A_{0}$, and drop those which do. The change in the coefficients is computed in [5]. After this approximation, the classical thermodynamics and the dimensionally reduced thermodynamics coincide exactly. The final form of the partition function describing the thermodynamics is then

$$
\begin{aligned}
Z & =\int \mathcal{D}\left(A_{i}, \Phi\right) \exp (-H / T) \\
H & =\int d^{3} x\left[\frac{1}{4 g^{2}} F_{i j}^{a} F_{i j}^{a}+\left(D_{i} \Phi\right)^{\dagger}\left(D_{i} \Phi\right)+m_{H T}^{2} \Phi^{\dagger} \Phi+\lambda\left(\Phi^{\dagger} \Phi\right)^{2}\right] .
\end{aligned}
$$

If this final integration over the $A_{0}$ field is not deemed reliable enough it is straightforward to modify what we will do below to include it in the thermodynamic calculation.

We will also mention a few results, perturbative and nonperturbative, which have been obtained for the partition function shown above. Perturbatively we can describe the strength of the phase transition by studying the effective potential for the (gauge fixed) Higgs field

$\phi, \phi=\sqrt{2 \Phi_{\text {cond }}^{\dagger} \Phi_{\text {cond }}}$. Note that there is no good gauge invariant, nonperturbative way to separate the condensate from the fluctuations. When gauge field fluctuations become large, the whole perturbative approach becomes questionable. Nevertheless, when the phase transition is strong, perturbation theory is useful, essentially because gauge field fluctuations are suppressed in the broken phase (which is therefore well described). At one loop, and neglecting scalar loops as is appropriate in the $\lambda / g^{2} \ll 1$ approximation,

$$
V_{1 \text { loop }}=\frac{m_{H T}^{2}}{2} \phi^{2}-\frac{g^{3}}{16 \pi} \phi^{3} T+\frac{\lambda}{4} \phi^{4} .
$$

This effective potential can have two minima because of the (one loop) negative $\phi^{3}$ term. Since it is a loop effect which allows a first order phase transition, we say the transition is radiatively induced first order. At the transition, the broken phase value of $\phi$ is such that the cubic term and (tree level) $\lambda \phi^{4}$ term are of the same order. Since this requires a one loop effect to be of the same size as a tree level one, it either implies that $\lambda / g^{2} \ll 1$, or that perturbation theory will not be a reliable expansion. Hence perturbation theory determines attributes of the transition at best as an expansion in $\lambda / g^{2}$. It is this relatively poor performance for perturbation theory which makes a nonperturbative treatment necessary.

Nonperturbatively it is known that, as expected, the phase transition is well described by perturbation theory for small $\lambda / g^{2}$; but perturbation theory is completely wrong for larger values [3,77. In fact there is NO phase transition in the MSM above a critical value $\lambda / g^{2} \simeq 0.0983 \pm 0.0015$ [8]. In extensions of the standard model with new bosons which are light at the phase transition, we must include the new light bosons in the effective theory considered. At least for the case of an added scalar top, the strength of the phase transition is significantly enhanced [33,34. It would be straightforward but more numerically expensive to apply the tools developed here to this physically interesting case.

\section{B. Dynamics: classical effective theories}

The infrared thermodynamics of the $\mathrm{SU}(2)$ sector of the standard model match those of classical 3+1 dimensional SU(2) Higgs theory, as discussed above. Does this matching also 
apply at the dynamical level, as originally conjectured (in a $1+1$ dimensional context) by Grigoriev and Rubakov [35]? In other words, are the dynamics of the infrared SU(2) Higgs fields described by classical Hamiltonian dynamics?

The answer is "no, it is more complicated than that." 1 As we now discuss, the dynamics of infrared gauge and Higgs fields are indeed classical; but they are not described by classical Hamiltonian dynamics. They are, to leading order in the logarithm of $g$, described by "classical" Langevin dynamics. Because a factor of 2 error in the treatment of the dynamics will change our determined rate by $\sim \exp ( \pm 1)$, while the rate itself is $\sim \exp (-100) T^{4}$, we will find it sufficient to make this approximation and take the dynamics to be Langevin.

To see that the dynamics of the real system are not classical Hamiltonian dynamics, look again at the thermodynamic discussion, especially Eq. (3.4) and Eq. (3.6). We see that there are linear UV divergences in the classical Debye mass for the $A_{0}$ field; and yet the $A_{0}$ field is related to the electric fields $E$, which generate the Hamiltonian dynamics for the gauge fields. This implies that there are divergent UV corrections to the gauge field dynamics of the classical fields. As first advocated by Bödeker, McLerran, and Smilga [36], we should consider this a potential problem for the study of the classical field dynamics. In fact, as argued by Arnold, Son, and Yaffe [37], what it means is that the classical Hamiltonian dynamics do not have a good regulation independent limit. Hence, the infrared gauge field dynamics of the classical theory technically do not exist. The dynamics of the full theory can scarcely coincide with those of the classical theory if the classical theory's dynamics are sick.

The physical origin of this problem is actually well known plasma physics. Transverse electric fields in a plasma feel Landau damping. This leads to very slow, overdamped evolution of infrared magnetic fields, as is typical in a conducting medium. As the classical theory cutoff is lifted, there are more and more "plasma" degrees of freedom, and the damping becomes ever more efficient. The correct treatment is to make the damping have the same efficiency as in the quantum theory. This requires studying the classical theory with hard thermal loop (HTL) effects [38] included. (The hard thermal loops are the nonabelian generalization of Debye screening, Landau damping, and other plasma effects familiar from electromagnetic plasmas.) Such a classical, but HTL including, treatment should be correct at leading order in the coupling $g$. Two numerical implementations of such a classical theory now exist; one [39] is based on a proposal by $\mathrm{Hu}$ and Müller [40, and one [17] is based on a proposal by Bödeker, McLerran, and Smilga [36], and more recently discussed by Iancu [41]. Both are extremely complicated. Probably the second method could be utilized in the type of computation we are going to discuss, but the numerical effort would be substantially greater than what we discuss below.

As first demonstrated by Bödeker [15], and further discussed and clarified both by Bödeker [42], Arnold, Son, and Yaffe [43], and Litim and Manuel 44, inclusion of the

\footnotetext{
${ }^{8}$ In defense of Grigoriev and Rubakov we should mention that the complications discussed in this subsection do not arise in $1+1$ dimensions, where the UV behavior is much more mild; hence their conjecture is correct for the problem they were addressing, namely the dynamics of the $1+1$ dimensional abelian Higgs model. The problem is applying it to the $3+1$ dimensional problem of interest instead.
} 
hard thermal loops in the infrared equations of motion is actually unnecessary, because at leading order in $\log (1 / g)$ the dynamics of the gauge fields are simple Langevin dynamics. We will not attempt to reproduce their arguments in detail here, but will only physically motivate them.

First, consider the behavior of an abelian plasma. We will distinguish two characteristic length scales: the Debye length $l_{\mathrm{D}} \sim 1 / m_{\mathrm{D}} \sim 1 / g T$, and the scattering length $l_{\text {scatt }} \sim$ $1 / g^{4} T \log (1 / g)$. The former is the shortest length scale where plasma effects are important. The latter is the mean length over which a current can propagate, before it is disrupted by collisions in the plasma. It coincides with the free path of a charge carrier to undergo large angle scattering.

On scales much longer than $l_{\text {scatt }}$, a magnetic field evolves as if it were in a conductor: $D \times B=j=\sigma_{\mathrm{el}} E=\sigma_{\mathrm{el}} D_{0} A$, where $\sigma_{\mathrm{el}}$ is the electric conductivity. This is just Langevin dynamics; the time derivative of the field $D_{0} A$ is proportional to $-d H / d A=D \times B$. In a thermal bath there is also a "noise" term uniquely determined by the thermodynamics (fluctuation dissipation). On scales between $l_{\mathrm{D}}$ and $l_{\text {scatt }}$ the story is significantly more complicated because the homogeneity scale is shorter than the free path over which an electric current propagates. In this regime the field evolution is described by an equation with a wave number dependent conductivity, which is nonlocal in real space.

Now consider the case of a nonabelian theory. The key difference is that, for the nonabelian theory, $l_{\text {scatt }} \sim 1 / g^{2} T \log (1 / g)$. The reason is that nonabelian collisions exchange nonabelian charge ("color"), so any collision, however soft, can destroy the current a particle is carrying. Hence $l_{\text {scatt }}$ is of order the mean free path for any scattering, not just large angle scattering. Hence, on the length scale $1 / g^{2} T$, the dynamics are given by a simple Langevin equation [18],

$$
\sigma_{\mathrm{el}} D_{0} A_{i}=-\frac{\partial H}{\partial A_{i}}, \quad \sigma_{\mathrm{el}}^{-1}=\frac{3}{m_{D}^{2}} \gamma, \quad \gamma=\frac{N_{\mathrm{c}} g^{2} T}{4 \pi}\left[\ln \frac{m_{\mathrm{D}}}{\gamma}+3.041\right],
$$

with $N_{c}=2$ for our $\mathrm{SU}(2)$ application. Here $\sigma_{\mathrm{el}}$ is the nonabelian ("color") conductivity.

The extension of these ideas to the case where there is a Higgs field turns out to be remarkably simple; one also evolves the Higgs fields under Langevin dynamics, but giving the Higgs fields a much larger diffusion constant than the gauge fields. For a discussion see [19]. These are the dynamics we will apply for the real time part of our studies below. Note that they are only justified at (next to [18]) leading order in $1 / \log (1 / g)$, not a very good expansion; but we are willing to accept an approximation which will yield an error of \pm 1 in the exponent of the nucleation rate, since the rate itself is $\sim \exp (-100)$.

\section{COMPUTATIONAL DETAILS AND NUMERICS}

\section{A. Our choice for a measurable}

In the discussion above we always chose to consider $\phi_{\mathrm{av}}^{2}$, the space averaged Higgs field length squared, as the observable used to distinguish the phases and the critical bubble. This has been the traditional "order parameter" observable in Monte Carlo simulations of SU(2) + Higgs theory. It is easy to measure, and, because its variance is UV finite, given large 
enough volume, it can unambiguously separate the symmetric and broken phases. Also, as we have seen, it is an extremely convenient choice because it makes it quite easy to use one set of multicanonical data to study a range of temperatures. Further, after UV counterterm subtractions, it has a good zero lattice spacing limit.

However, a priori it is not obvious that this (or almost any other) measurable can distinguish the critical bubble well enough for practical calculations. In particular we might worry that there is too large a "noise" contribution from the $85 \%$ of the volume which must be in the symmetric phase (see the discussion in subsection एव). A necessary (but insufficient) criterion for a good measurable, needed to avoid this problem, is that it has a small variance in the symmetric phase. It turns out that $\phi_{\mathrm{av}}^{2}$ does indeed have a very small variance in the symmetric phase. The leading order perturbative result for the variance of $\phi_{\mathrm{av}}^{2}$ in the symmetric phase, in a volume $V$ much larger than $1 / m_{\text {symm }}^{3}$, with $m_{\text {symm }}$ the symmetric phase scalar mass, is

$$
\begin{aligned}
\sigma_{\phi_{\mathrm{av}}^{2}, \mathrm{symm}}^{2} & =\frac{1}{V^{2}} \int d^{3} x d^{3} y\left\langle\phi^{2}(x) \phi^{2}(y)\right\rangle_{\text {connected }} \\
& =\frac{4}{V} \int \frac{d^{3} p}{(2 \pi)^{3}} \frac{T^{2}}{\left(p^{2}+m^{2}\right)^{2}}=\frac{T^{2}}{2 \pi m V} \simeq \frac{4 T \sqrt{2 \lambda / g^{2}}}{g^{2} V},
\end{aligned}
$$

where in the last approximate equality we have substituted in the equilibrium, one loop symmetric phase Higgs mass for $m$. This variance is to be compared to the broken phase variance, which gets an added contribution from fluctuations in the zero mode, which has a condensate $\phi_{0}$ :

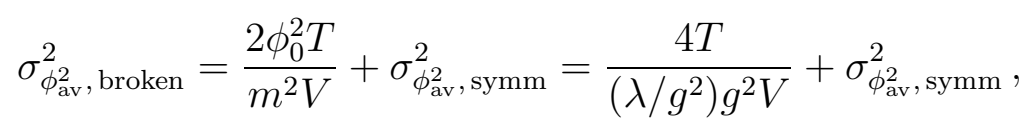

which is much larger for small $\left(\lambda / g^{2}\right)$. To get a phase transition strong enough to preserve baryon number after its conclusion, we will consider the case $\lambda / g^{2}=0.036$; for this case the broken phase variance is about 100 times larger, and if $15 \%$ of the volume is in the broken phase, the variance contributed by this volume greatly exceeds that contributed by the symmetric phase; symmetric phase fluctuations will not pose a problem. Also note that the broken phase fluctuations are dominated by the motion of the condensate; and we expect that fluctuations in the condensate size are directly important to whether a bubble is more or less than critical, so these fluctuations may also not be dangerous.

A posteriori we will of course determine whether the choice of measurable was a good one. For instance, when determining $\mathbf{d}$, we can determine what fraction of trajectories crossing $\phi_{\mathrm{av}}^{2}=\phi_{\mathrm{av}, \mathrm{C}}^{2}$, actually lead to a nucleation. We will find that $\phi_{\mathrm{av}}^{2}$ is in fact a good measurable; the fraction of trajectories crossing the critical bubble which lead to a nucleation is statistically compatible with $1 / 2$, which is the maximum possible under Langevin dynamics. Note however that the above arguments suggest that, if we were studying nucleation out of the broken phase, then $\phi_{\mathrm{av}}^{2}$ would probably be a very bad measurable. We will not discuss what measurables might be useful for studying this nucleation rate, which fortunately is not cosmologically interesting.

Note that the value for the scalar self-coupling we use, produces a Higgs mass lighter than the experimental limit; in fact there is no physical Higgs mass which gives such a small 
value for the ratio of $\lambda / g^{2}$ (parameters of a 3-D effective theory) [5]. We study this case as a toy example, because the phase transition is relatively strong here and perturbation theory arguably should be reasonable.

\section{B. Multicanonical method}

As described in Sect. II, by far the dominant factor in the bubble nucleation rate is determined by the constrained free energy $F\left(\phi_{\mathrm{av}}^{2}\right)=-T \log P_{\text {can. }}\left(\phi_{\mathrm{av}}^{2}\right)$, where $P_{\text {can. }}\left(\phi_{\mathrm{av}}^{2}\right)$ is the canonical probability distribution of $\phi_{\mathrm{av}}^{2}$ for a particular value of $m_{H T}^{2}$ :

$$
P_{\text {can. }}\left(\phi_{\mathrm{av}}^{2}\right) \propto \int \mathcal{D}\left(A_{i}, \Phi\right) \exp [-H / T] \delta\left(\phi_{\mathrm{av}}^{2}-\sum_{x} 2 \Phi^{\dagger} \Phi / V\right) .
$$

(The 2 next to $\Phi^{\dagger} \Phi$ is for the customary complex normalization of the Higgs field.) This probability distribution has to be determined for the whole range of values from the symmetric phase to somewhat beyond the critical bubble value $\phi_{\mathrm{av}, C}^{2}$, see Fig. 3 .

In principle, $P_{\text {can. }}\left(\phi_{\text {av }}^{2}\right)$ can be calculated with a standard lattice Monte Carlo computation, where the configurations are sampled with the canonical probability

$$
p_{\text {can. }} \propto \exp (-H / T)
$$

where $H$ is given in Eq. (3.7). Algorithms for such a sampling are well known, typically taking the form of a Markov chain in which each configuration is a relatively small modification of the previous one.

However, in the problem we are interested in, the probability can vary by a factor of $\sim \exp (100)$ over the range of $\phi_{\mathrm{av}}^{2}$ of interest. A finite, canonical sample will simply contain no representatives for much of the range of $\phi_{\mathrm{av}}^{2}$ of interest, and hence give no information on the free energy in that part of the range; hence it will fail to determine $P_{\text {can. }}$. For our problem, the canonical Monte Carlo method is utterly useless. This is exactly the kind of problem where multicanonical Monte Carlo methods excel.

In a multicanonical simulation, the Monte Carlo sampling probability of configurations is modified so that the whole $\phi_{\mathrm{av}}^{2}$ range of interest is sampled with an approximately constant probability. This is achieved by sampling the configurations according to the probability

$$
p_{\text {muca }} \propto \exp \left[-H / T+W\left(\phi_{\mathrm{av}}^{2}\right)\right],
$$

where the weight function $W\left(\phi_{\mathrm{av}}^{2}\right)$ is carefully tuned so that the multicanonical probability distribution

$$
\begin{aligned}
P_{\text {muca }}\left(\phi_{\text {av }}^{2}\right) & \propto \int \mathcal{D}\left(A_{i}, \Phi\right) \exp [-H / T+W] \delta\left(\phi_{\text {av }}^{2}-\sum_{x} 2 \Phi^{\dagger} \Phi / V\right) \\
& \propto \exp \left[W\left(\phi_{\text {av }}^{2}\right)\right] P_{\text {can. }}\left(\phi_{\text {av }}^{2}\right)
\end{aligned}
$$

is approximately constant. This condition is met if

$$
W\left(\phi_{\mathrm{av}}^{2}\right) \approx-\log P_{\mathrm{can} .}\left(\phi_{\mathrm{av}}^{2}\right)+\text { const. }
$$


The canonical expectation value of any observable $\mathcal{O}$ can then be obtained from a multicanonically sampled set of configurations by reweighting the individual measurements with the weight function:

$$
\langle\mathcal{O}\rangle=\sum_{k} \mathcal{O}_{k} e^{-W\left(\phi_{\mathrm{av}, k}^{2}\right)} / \sum_{k} e^{-W\left(\phi_{\mathrm{av}, k}^{2}\right)},
$$

where the sums go over all configurations in the sample. It is not difficult to find an algorithm to perform the multicanonical update; if one has a Markovian canonical Monte Carlo algorithm, application of Metropolis accept-reject under the weight function $\exp \left(W\left(\phi_{\mathrm{av}}^{2}\right)\right)$ after each update yields a multicanonical algorithm. As $\phi_{\mathrm{av}}^{2}$ is an easy observable to measure numerically, the numerical cost of this extra step is negligible. (However, since $\phi_{\mathrm{av}}^{2}$ is a global quantity, some extra work is needed when using parallel computer architectures.)

From Eqs. (4.6) and (4.7) we see the main difficulty of the multicanonical method; we have to know the result we are after, $P_{\text {can. }}$, to some accuracy, before we can even start the multicanonical simulation! This requires some kind of bootstrap process, to be discussed below, in order to determine an initial guess for $P_{\text {can. }}$; after the multicanonical simulation, we get an improved estimate for $P_{\text {can. }}$ from Eq. (4.6).

In the above discussion we implicitly assumed that the weight function $W$ has been optimized for one particular value of $m_{H T}^{2}$. However, due to the factorization property of the $m_{H T}^{2}$ term in the Hamiltonian, Eq. (2.5), we obtain the canonical probability $P_{\text {can. }}$ for a whole range of $m_{H T}^{2}$ values from a single multicanonical Monte Carlo run: for example, if the multicanonical weight function has been originally calculated with $m_{H T}^{2}=m_{1}^{2}$ (and we have the resulting distribution $P_{\text {muca }}$ ), we have

$$
P_{\text {can. }}\left(m_{2}^{2} ; \phi_{\mathrm{av}}^{2}\right) \propto P_{\mathrm{muca}}\left(\phi_{\mathrm{av}}^{2}\right) \exp \left[-W\left(\phi_{\mathrm{av}}^{2}\right)+\frac{V}{2 T}\left(m_{1}^{2}-m_{2}^{2}\right) \phi_{\mathrm{av}}^{2}\right] .
$$

Naturally we can only determine $P_{\text {can. }}\left(m^{2} ; \phi_{\text {av }}^{2}\right)$ for values of $\phi_{\text {av }}^{2}$ where our $P_{\text {muca }}$ determination is accurate. Strictly speaking, when we perform a multicanonical run it does not correspond to any particular value of $m^{2}$, since we can always absorb the $m^{2}$ term in the action into the weight function.

How does the computational cost scale in a multicanonical simulation? Ideally, if we have guessed $W\left(\phi_{\mathrm{av}}^{2}\right)$ correctly, the system performs a random walk in the $\phi_{\mathrm{av}}^{2}$ range of interest, say, from $\phi_{\mathrm{av} 1}^{2}$ to $\phi_{\mathrm{av} 2}^{2}$. Let us consider what happens if keep the range of $\phi_{\mathrm{av}}^{2}$ considered fixed as we increase the volume of the system. Now, if we require that the system "random walks" through the range a comparable number of times as the volume is increased, the computational cost is proportional to $\left(\phi_{\mathrm{av} 2}^{2}-\phi_{\mathrm{av} 1}^{2}\right)^{2} V^{2}$. The factor $V^{2}$ appears because $\phi_{\mathrm{av}}^{2}$ is an intensive variable. For comparison, in a canonical simulation at a first order phase transition, the numerical cost rises as $\max _{\phi_{\mathrm{av}}^{2}}\left(P_{\text {can. }}\left(\phi_{\mathrm{av}}^{2}\right)\right) / \min _{\phi_{\mathrm{av}}^{2}}\left(P_{\text {can. }}\left(\phi_{\mathrm{av}}^{2}\right)\right)$. In a large box, as previously discussed, this scales as the exponential of an interface surface area, $\sim \exp \left(2 \sigma V^{2 / 3}\right)$, which is a vastly more severe increase.

In realistic situations there are often "hidden" barriers, which can hinder the random walk through the range of interest. For instance, in our case such a metastability occurs in large cubic volumes when the surface geometry changes: as shown in Fig. 4 , there can be sphere $\leftrightarrow$ cylinder and cylinder $\leftrightarrow$ slab transitions. At the transition point two different geometries have equal volume fractions and surface area. During a simulation the transition must occur 
by the Monte Carlo algorithm finding a series of mixed phase geometries which smoothly interpolate between cylinder and sphere, which means that the surface area must increase for a fixed volume fraction. Thus, if we use extremely large volumes, the transitions between geometries become exponentially suppressed, even if the total multicanonical probability remains constant. (Of course for the study of bubble nucleation we will not have to study the range of $\phi_{\mathrm{av}}^{2}$ where such transitions occur; but when we determine $T_{\mathrm{eq}}$, or measure the surface tension (see below), it can become an issue. We also observe some metastability at the value of $\phi_{\mathrm{av}}^{2}$ where the dominant configuration changes from being homogeneous symmetric phase to a small broken phase bubble.)

The success of the multicanonical method hinges on the accurate determination of the weight function. If we require that the resulting probability distribution $P_{\text {muca }}$ is constant up to factor of 2, say, then the weight function must be determined up to an accuracy of $\log (2) \approx 0.7$. Worse accuracy in determining $W$ significantly degrades the efficiency of the subsequent Monte Carlo, so such a requirement on the accuracy of $W$ is actually necessary. The variation of $W$ across the range of interest in this work is of order $\approx 100$ (that is, we have to boost the probability of suppressed phase space regions by a factor of $\exp (100)$.) Thus, the weight function has to be determined to an overall accuracy better than $1 \%$.

We use a continuous, piecewise linear Ansatz for the weight function. We determine the weight function with an automatic iterative calculation procedure, using variations of the procedures presented in [34, 30]. One approach is to choose a starting guess for $W\left(\phi_{\mathrm{av}}^{2}\right)$ (for instance, a constant), and to perform a Monte Carlo under the (Markov chain) algorithm which would generate the distribution, Eq. (4.5). However, after each update sweep, $W$ is decremented at the current value of $\phi_{\mathrm{av}}^{2}$. Thus, if some region of $\phi_{\mathrm{av}}^{2}$ is getting sampled very often, $W$ is reduced there so it will be sampled less often. This procedure will cause $W$ to evolve towards the correct form, but imperfectly because the recent history of the Monte Carlo is over-reflected in the resulting $W$. To fix this, the size of the decrements is reduced every time the evolution successfully explores the full range of $\phi_{\mathrm{av}}^{2}$ from bottom to top and back. When the total change to $W$, in the time the Monte Carlo evolution spans the range of $\phi_{\mathrm{av}}^{2}$, is negligible, the weight function has been determined with sufficient accuracy. Measurements of $P_{\text {can. }}$ then consist of two parts: first, we perform a run during which the weight function is iteratively improved to a required accuracy. Second, using this weight function, we perform a normal multicanonical run, which gives us the final probability distribution. The determination of $W$ typically accounts for $30-50 \%$ of the total computational effort.

\section{An application: surface tension}

We illustrate how the multicanonical method works by measuring the surface tension $\sigma$ - i.e. the free energy/area carried by the phase interface - with the histogram method [45]. This has become the standard and well-understood method for computing the surface tension in a variety of lattice theories, including work closely related to ours, $\mathrm{SU}(2)$ gauge + Higgs theories [3,46.9] and effective theories for the MSSM [34].

As discussed in Sect. П1. at the phase transition value of $m_{H T}^{2}$, where the symmetric and broken phases are equally probable, the mixed phase configurations with approximately equal volume fractions of symmetric and broken phases are exponentially suppressed (Fig. 2). 
The suppression is proportional to $\exp \left(-F_{\text {surface }} / T\right)=\exp (-\sigma \times$ Area $/ T)$. This is seen as a valley in the probability distribution of $\phi_{\mathrm{av}}^{2}$, see Fig. 6.

For the interface tension measurements it is advantageous to use lattices with cylindrical geometry, $L_{z} \gg L_{x}=L_{y}$. Because we use periodic boundary conditions, there will be at least two interfaces which span the lattice. The cylindrical geometry makes the interfaces tend to form parallel to the $(x, y)$-plane; and $L_{z}$ should then be long enough so that the two interfaces do not interact appreciably. This is seen as a flat minimum in the probability distributions.

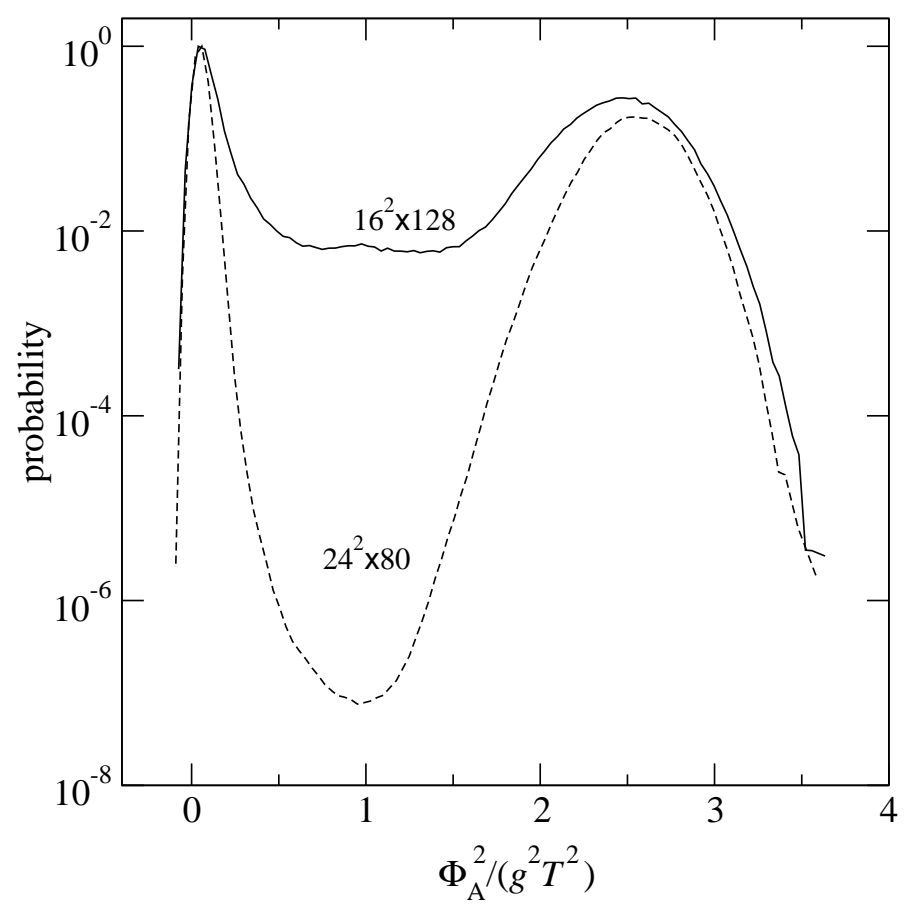

FIG. 6. Probability distributions of $\phi_{\mathrm{av}}^{2}$ measured from cylindrical lattices at $\beta=4 /\left(g^{2} T a\right)=9$ and $\lambda / g^{2}=0.036$.

We perform a multicanonical Monte Carlo with the same lattice action and update as [3], using the improved relation between lattice and continuum parameters found in [6]. In Fig. 6 we show the probability distributions from $16^{2} \times 128$ and $24^{2} \times 80$ lattices at $4 /\left(g^{2} \mathrm{Ta}\right)=9$ and $\lambda / g^{2}=0.036$, P measured at the critical $m^{2}$, which is determined by requiring that the symmetric and broken phases have equal probabilistic weight. (Hence, the technique also provides an accurate determination of the the critical value of $\mathrm{m}^{2}$, which we will need for comparison later on.) The larger lattice is not quite long enough to have the flat central part the smaller lattice has. Note the striking difference in widths between the symmetric (left) and broken (right) phase peaks; this reflects the large ratio in the $\phi_{\mathrm{av}}^{2}$ variance in the two phases, discussed in subsection $\mathbb{I V A}$.

\footnotetext{
${ }^{9}$ Corresponding to "unimproved" lattice $\beta_{G}=9.6674, x \equiv \lambda / g^{2}=0.0389$. For the lattice to continuum relations for $m^{2}$ and $\phi^{2}$ see [6].
} 


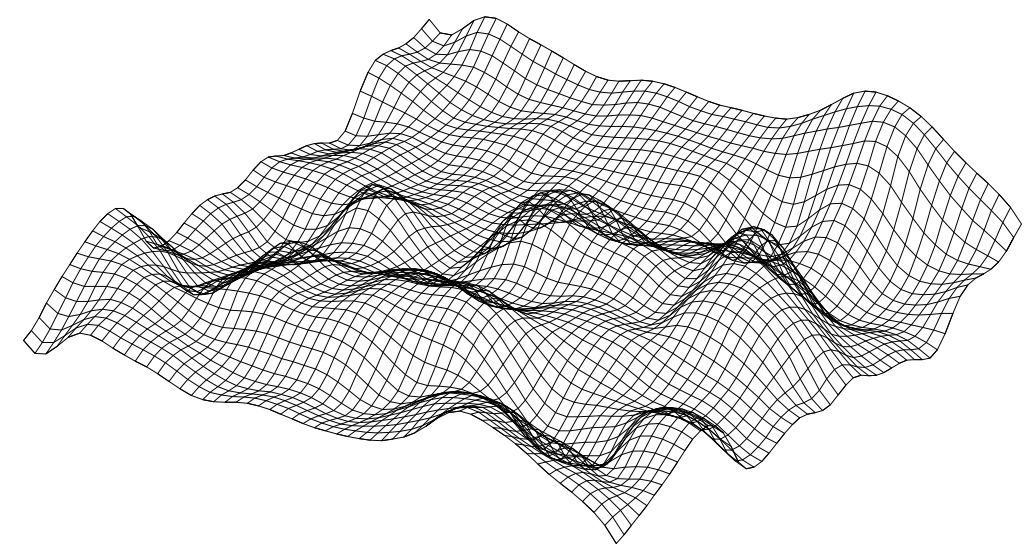

FIG. 7. Geometric shape of a phase interface, from a $62 / g^{2} T$ across box. The height of the fluctuations goes as $(T / \sigma)^{1 / 2}$; Fourier analyzing and averaging over hundreds of such surfaces can yield an accurate determination of $\sigma$.

\begin{tabular}{|c|c|c|}
\hline Spacing $a / g^{2} T$ & Volumes used & $\sigma$ \\
\hline $4 / 7$ & $72^{2} \times 96,80^{2} \times 120,108^{2} \times 144$ & $(0.0749 \pm 0.0008) g^{4} T^{3}$ \\
\hline $4 / 9$ & $60^{2} \times 144,96^{2} \times 144,108^{2} \times 160$ & $(0.0758 \pm 0.0014) g^{4} T^{3}$ \\
\hline $4 / 12$ & $120^{2} \times 160,132^{2} \times 180$ & $(0.0733 \pm 0.0025) g^{4} T^{3}$ \\
\hline
\end{tabular}

TABLE II. Surface tension as a function of lattice spacing.

The surface tension is obtained from $\log \left[P_{\max } / P_{\min }\right] /\left(2 L_{x}^{2}\right) \rightarrow \sigma / T$, as $V \rightarrow \infty$. In practice, the infinite volume value of $\sigma$ is reached in such large volumes that finite volume analysis becomes necessary. Following Ref. 47,48, we fit the data with the Ansatz which takes into account the translation modes of the surfaces and capillary fluctuations:

$$
\frac{\sigma}{T} a^{2}=\frac{1}{2\left(L_{x} a\right)^{2}}\left[\log \frac{P_{\max }}{P_{\min }}+\frac{3}{2} \log L_{z} a-\log L_{x} a+\text { const. }\right] .
$$

The result of the fit from these two lattices is

$$
\sigma=(0.079 \pm 0.004) g^{4} T^{3}
$$

We also obtain the equilibrium $\mathrm{m}^{2}$, and the difference in $\phi_{\mathrm{av}}^{2}$ between the two phases, which in physical units is $\Delta \phi_{\mathrm{av}}^{2} \equiv \phi_{\mathrm{av}}^{2}($ broken $)-\phi_{\mathrm{av}}^{2}(\mathrm{symm})=2.53 g^{2} T^{2}$.

The surface tension can be obtained much more economically with an alternative method due to Moore and Turok 49. This method is based on analyzing the spectrum of the transverse fluctuations of the phase interfaces; the magnitude of the fluctuations is inversely proportional to $\sqrt{\sigma / T}$. We refer the reader to the reference for a complete discussion. We apply this method using multicanonical tools to sample configurations in a very large box, but now choosing $W\left(\phi_{\mathrm{av}}^{2}\right)$ to very strongly prefer $\phi_{\mathrm{av}}^{2}$ within $5 \%$ of the average between symmetric and broken values; hence the volume always contains large regions of each phase, with two approximately planar interfaces separating them. We show an example of such an interface in Fig. 7, and present the determined surface tension, as a function of lattice spacing, in Table II]. The results agree within error with the histogram method. If we 
extrapolate the values given in the table to $a \rightarrow 0$ assuming $O\left(a^{2}\right)$ errors, as should be the case since we use an $O(a)$ corrected lattice-continuum match, we obtain the result

$$
\sigma=(0.0749 \pm 0.0027) g^{4} T^{3}, \quad a=0,
$$

with a lattice spacing dependence which is small and consistent with zero. This indicates that our lattice spacing errors are under control.

\section{Results: probability distribution}

Let us now turn to our main problem, the determination of the probability distribution in the region relevant to bubble nucleation, see Fig. 3. The procedure is very similar to the surface tension calculation with the histogram method described above. However, there are two crucial points where it differs: 1) we need very large, preferably cubical volumes in order for the bubbles to fit in the lattice comfortably. The size makes it next to impossible to compute the full probability distribution from the symmetric to the broken phase with our computational resources. However, 2) we need the weight function only in the range $\phi_{\mathrm{av}}^{2}(\mathrm{symm}) \leq \phi_{\mathrm{av}}^{2}<\left(0.85 \phi_{\mathrm{av}}^{2}(\mathrm{symm})+0.15 \phi_{\mathrm{av}}^{2}\right.$ (broken $\left.)\right)$, as discussed in Sect. II . This guarantees that we do not yet enter the "cylinder" and "slab" regions of the phase space, see Fig. 1 . Using the random walk argument, calculating the distribution in this restricted range only requires a factor of $(0.15)^{2} \approx 0.02$ of the resources needed for the full weight function. 10

In Fig. 8 we show the probability distributions from a $124^{3}$ lattice, with $4 /\left(g^{2} T a\right)=$ 9. The result of a single multicanonical run has been reweighted to 3 different values of $m_{H T}^{2}$, namely $(0.008,0.009,0.010) g^{4} T^{2}$ below the equilibrium value. The critical bubbles correspond to the minimum locations of the probability. Note that the value of $\phi_{\mathrm{av}}^{2}$ for the critical bubble moves to smaller values as we increase the supercooling; this is because the critical bubble gets smaller at larger supercooling, and $\phi_{\mathrm{av}}^{2}$ is a volume average. We also have results from a $92^{3}$ box at $4 /\left(g^{2} T a\right)=7$, to study lattice spacing dependence, and from a $120^{3}$ box at $4 /\left(g^{2} T a\right)=9$, which gives weak information on volume dependence. The ratio of probabilities between the metastable minimum and the critical bubble, $P_{\text {can. }}\left(\phi_{\text {av }}^{2}=\right.$ $\left.\phi_{\mathrm{av}, \mathrm{C}}^{2}\right) / P_{\text {can. }}\left(\phi_{\mathrm{av}}^{2}=\phi_{\mathrm{av}}^{2}(\mathrm{symm})\right)$, is plotted for each lattice as a function of $\delta m^{2} \equiv m_{H T}^{2}-$ $m_{H T}^{2}$ (equilib) in Fig. 9. This figure corresponds to the difference in height, in Fig. \&, between the local maximum and local minimum. For each curve in the figure, the statistical error bars are about \pm 1 , with strong correlation in the error along the curve. The finer spacing lattices agree within errors. This is a (weak) check on volume dependence, but it is also a check of the code, since the $120^{3}$ and $124^{3}$ volume computations were performed with completely independent sets of code, on machines of different architecture. The coarser lattice data differs by between $2 \%$ and $3 \%$. This probably represents lattice spacing errors; if we extrapolate assuming $a^{2}$ errors (the first order which should be present, due to our lattice improvement), we estimate the difference between the finer lattice and the continuum

\footnotetext{
${ }^{10}$ This discounts the "barriers" at the bubble $\leftrightarrow$ cylinder $\leftrightarrow$ slab transitions, which would make the full computation even more costly.
} 


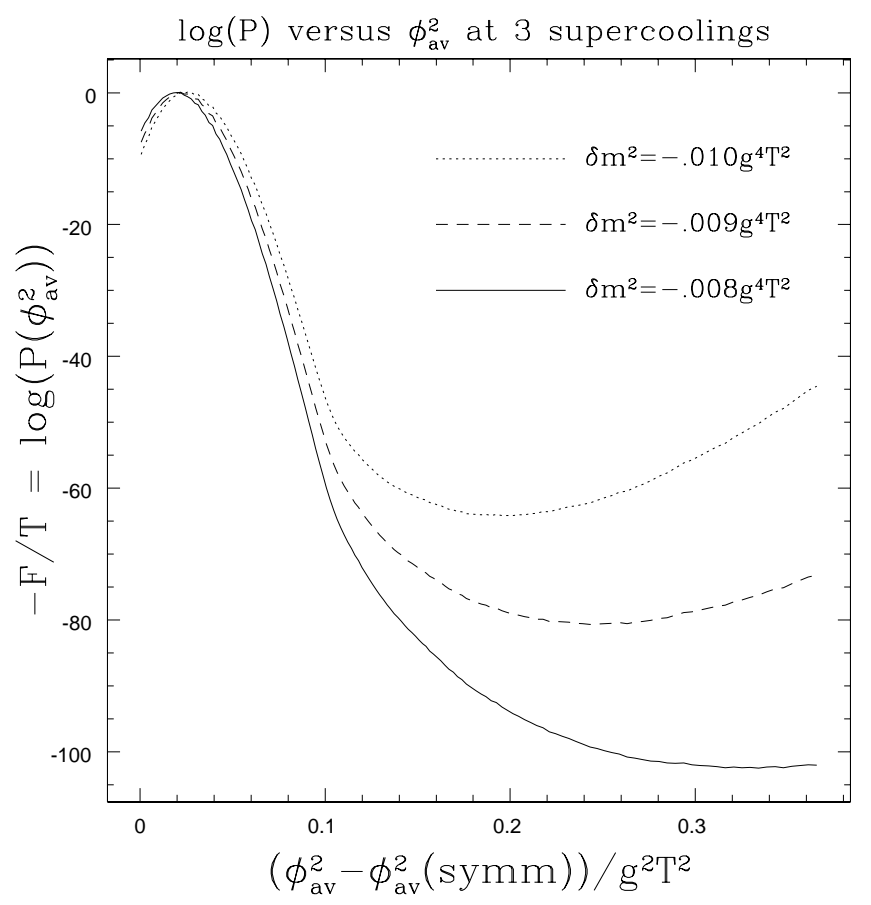

FIG. 8. The probability distribution for $\phi_{\mathrm{av}}^{2}$ at three values of $\delta m^{2},-0.008 g^{4} T^{2}$ (solid), $-0.009 g^{4} T^{2}$ (dashed), and $-0.010 g^{4} T^{2}$ (dotted), for $124^{3}$ lattice at $g^{2} a T=4 / 9$. In each case the local maximum is the supercooled symmetric phase, the local minimum is the critical bubble. The three curves are obtained by reweighting the same multicanonical run. Greater supercooling leads to less suppression of the critical bubble.

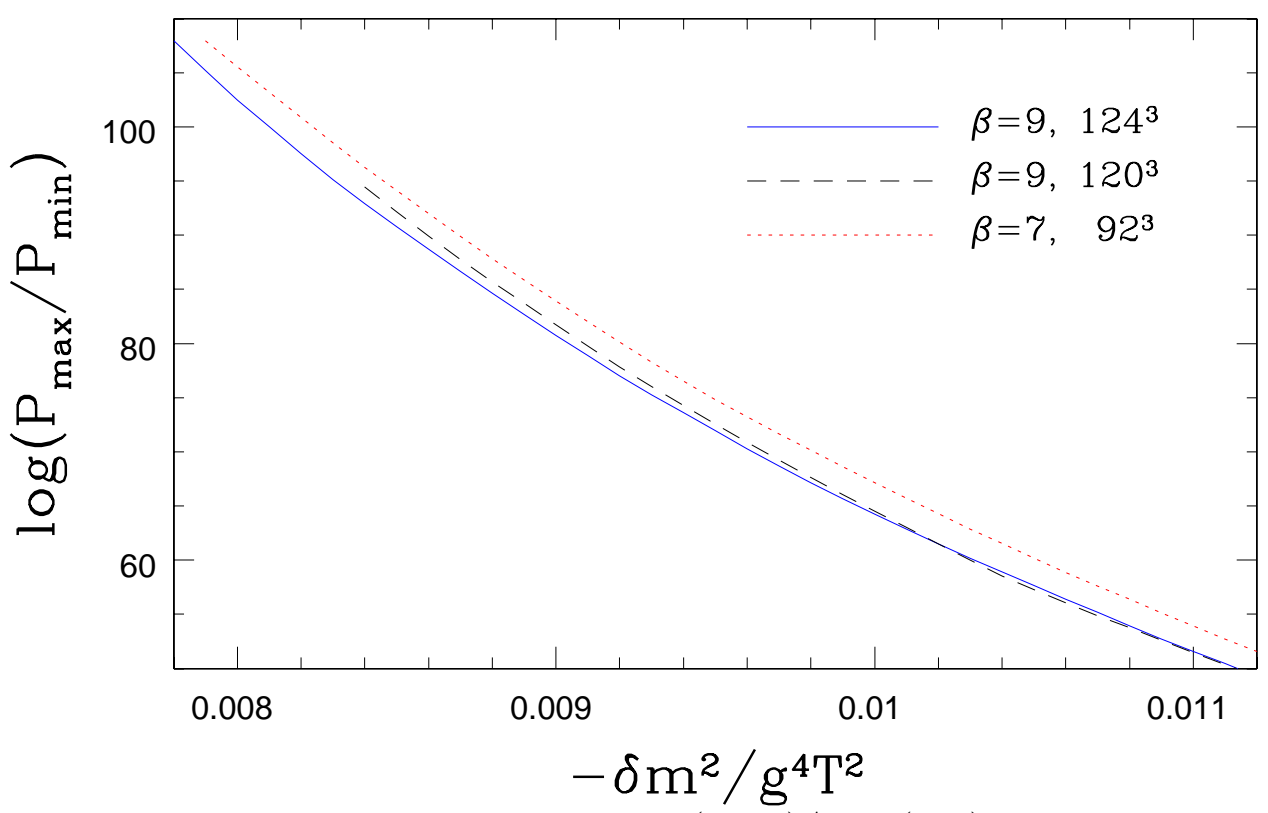

FIG. 9. Log of ratio of probabilities $\log P_{\text {can. }}$ (meta) $/ P_{\text {can. }}$ (crit), as a function of $\delta m^{2}$ the supercooling from the equilibrium $\mathrm{m}^{2}$. The solid line is the $124^{3}$ lattice, and the dashed line is the $120^{3}$ lattice, at $\left(4 / g^{2} T a\right)=9$. Each has a statistical error of \pm 1 , so they agree within expected error. The dotted line is the data from the $92^{3}$ lattice at $\left(4 / g^{2} T a\right)=7$. Its disagreement represents definite, but small, lattice spacing error. 
is 1.5 times as large as the difference in the curves. Where the ratio of probabilities is $e^{100}$, this would be a correction of $e^{4}$ in the nucleation rate, or about a $2.5 \%$ correction in $\delta m^{2}$. It remains to integrate the area in the symmetric phase minimum, to compute the dynamical contributions, and divide by the volume, to convert this result into the real time rate.

\section{E. Dynamical prefactor, tools and calculation}

To determine the real time rate for nucleations, we now have to perform real time evolution on each of a sample of configurations with $\phi_{\mathrm{av}}^{2}=\phi_{\mathrm{av}, \mathrm{C}}^{2}$. We get the sample of such configurations by, first, choosing a $\delta m^{2}$ to consider, and, next, by performing a multicanonical Monte Carlo, as just described, and recording those configurations for which $\phi_{\text {av }}^{2}$ lies within a narrow tolerance of $\phi_{\mathrm{av}, \mathrm{C}}^{2}$ (which depends on $\delta m^{2}$, see Fig. 8). In fact we can speed up the sampling process by choosing a weight function $W\left(\phi_{\mathrm{av}}^{2}\right)$ which favors $\phi_{\mathrm{av}}^{2}=\phi_{\mathrm{av}, \mathrm{C}}^{2}$ even more strongly than the one used to determine the probability distribution in the last section. Then, we must study the real time evolution of each configuration in the sample, at the thermal Higgs mass $m_{\mathrm{eq}}^{2}-\delta m^{2}$.

As discussed in subsec. IIIB, the appropriate dynamics, at leading log, are Langevin dynamics. The gauge fields evolve according to Eq. (3.9), and the Higgs fields also evolve under Langevin dynamics, with a much (parametrically) faster time scale. In continuum notation, this means evolving the fields under the following Langevin field equations (normalizing $E$ so $E^{2} / 2 g^{2}$ appears in the action),

$$
\begin{aligned}
\sigma_{\mathrm{el}} E_{i}^{a}(x) & =-\frac{\partial}{\partial A_{i}^{a}(x)} H+\xi_{i}^{a}(x, t), \\
\sigma_{\mathrm{el}} D_{t} \Phi(x) & =-\eta \frac{\partial}{\partial \Phi^{\dagger}(x)} H+\xi_{\Phi}(x, t), \\
\left\langle\xi_{i}^{a}(x, t) \xi_{j}^{b}\left(x^{\prime}, t^{\prime}\right)\right\rangle & =2 \sigma_{\mathrm{el}} T \delta_{i j} \delta^{a b} \delta\left(x-x^{\prime}\right) \delta\left(t-t^{\prime}\right), \\
\left\langle\xi_{\Phi}(x, t) \xi_{\Phi}^{\dagger}\left(x^{\prime}, t^{\prime}\right)\right\rangle & =2 \eta \sigma_{\mathrm{el}} T \mathbf{1} \delta\left(x-x^{\prime}\right) \delta\left(t-t^{\prime}\right),
\end{aligned}
$$

where $H$ is given in Eq. (3.7), 1 is the identity in component space for the Higgs field and $\eta$ is the ratio of the speeds of Langevin evolution, which is parametrically $\sim 1 / \alpha_{w}$, and so should be taken large.

It is possible to perform numerical Langevin evolution on lattice fields, but it is slow and unnecessary; any dissipative update will do, if the relation between the number of updates and Langevin time is known. Hence we use the heat bath algorithm to update the gauge fields; the relation between the number of heat bath updates, and the time scale $t$ in the equations above, is discussed at some length in [16]. At leading order in small $a$ the relation is that, for random order heat bath updates of the lattice links, $n$ updates per link corresponds to $\Delta t=a^{2} \sigma_{\mathrm{el}} n / 4$. (Note that this relation is specifically for our choice of lattice action, namely "Wilson glue"; it would be different for an improved action. It can also differs if the sites are updated in a specific, rather than random, order, and in fact depends on the order of update.) We update the Higgs fields with a mixture of the over-relaxation algorithm presented in [3] and a Higgs field heat bath algorithm. Note that our "real time" evolution algorithm can be viewed as a canonical Monte Carlo evolution algorithm at $m^{2}=m_{\text {eq }}^{2}-\delta m^{2}$; 


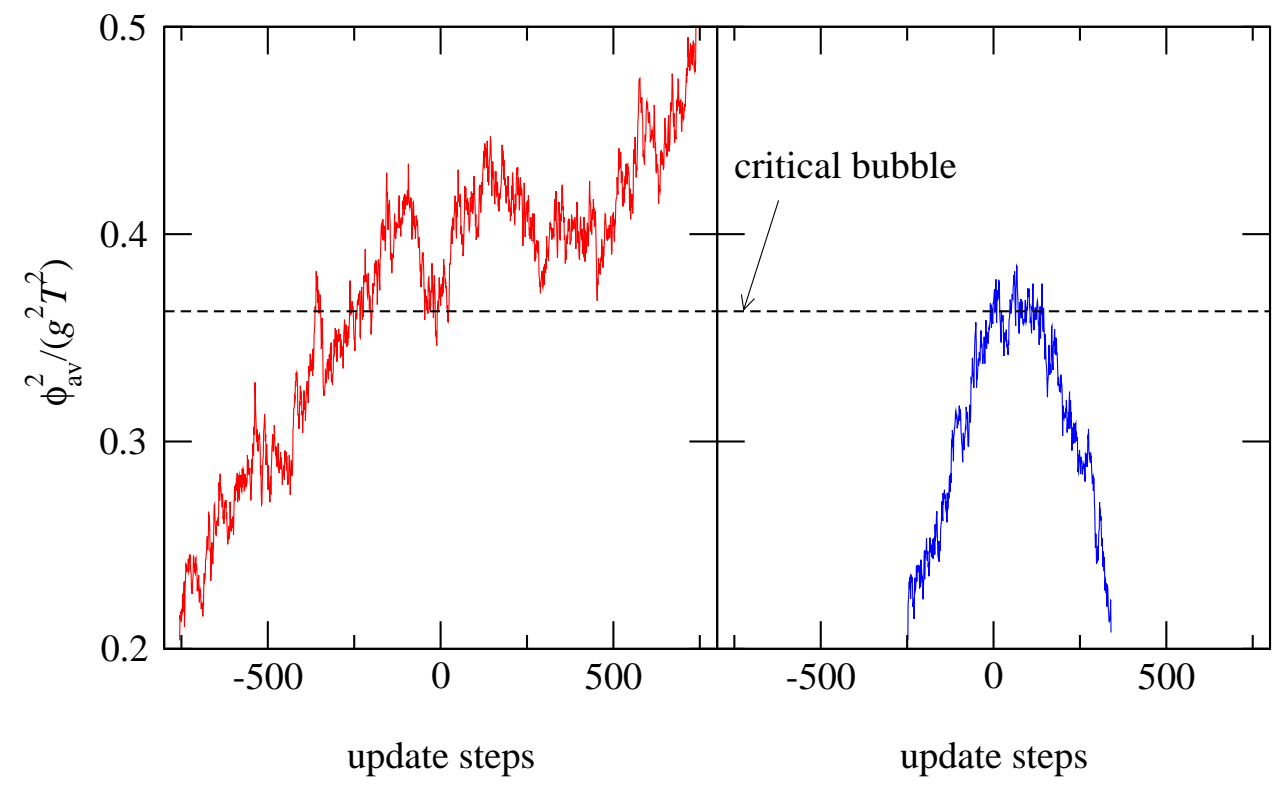

FIG. 10. A tunnelling trajectory and a trajectory which does not lead to tunnelling, measured from $120^{3}$ volume and at supercooling $\delta m^{2}=-0.0082 g^{4} T^{2}$. The horizontal dashed line is the critical bubble value of $\phi_{\mathrm{av}}^{2}$. Two half-trajectories (positive and negative timestep values) are evaluated starting from a configuration at the critical value of $\phi_{\mathrm{av}}^{2}$, and glued together at timestep 0 to form a full trajectory.

hence there is no concern that it somehow spoils the thermodynamics (as might happen for Langevin or Hamiltonian evolution with a finite time step, due to time step size errors).

As described in subsection [1B, we calculate the "dynamical prefactor" $\mathbf{d}$, Eq. (2.8), by evolving a critical bubble configuration both forward and backwards in time, long enough to see whether the system evolves either towards the symmetric or the broken phase. Since forward and backwards Langevin evolution are equivalent, in practice we generate a few Langevin trajectories from each initial critical bubble configuration; each pair can be joined together to form a full trajectory, see Fig. 10, and by considering all pairings we somewhat improve the statistics. The dynamical prefactor is then the expectation value

$$
\mathbf{d}=\frac{1}{N_{\text {traj. }}} \sum_{\text {traj. }} \frac{\delta_{\text {tunnel }}}{\# \text { of crossings }},
$$

where $\delta_{\text {tunnel }}$ is 1 if the trajectory leads to tunnelling, 0 otherwise, and (\# of crossings) is the number of times the trajectory crosses the critical bubble value of $\phi_{\mathrm{av}}^{2}$. When computing the error in the determination of $\mathbf{d}$ we must account for the dependence of the several trajectories involving a common critical bubble.

One legitimate concern is that the Langevin dynamics we consider are only correct at leading log, and as argued in [19], the treatment should break down when there is a large Higgs field condensate. Hence our treatment of the dynamics may not be better than an $O(1)$ treatment. However, our determination of the thermodynamic likelihood of a critical bubble was only good to \pm 1 in the exponent, so an $O(1)$ error in the dynamics is no worse; in any case, since the nucleation rate is $\sim e^{-100} T^{4}$, a factor of 2 error in its determination 
only represents a $1 \%$ error in the exponent, and somewhat less than a $1 \%$ error in the determination of $\delta m^{2}$, as seen in Fig. 9. It would be possible to do a better job by using the full HTL dynamics by the technique developed in [17]; however, this approach is much more numerically expensive. It also requires the inclusion of the $A_{0}$ field in the thermodynamic treatment, and it could be difficult to eliminate finite time step errors in the dynamics, which could make the thermodynamics explored by the evolution slightly incorrect.

The reader might also be concerned that the dynamics of a broken phase bubble will not have a good limit as $\eta$ is taken large. If the Higgs fields are allowed to evolve much faster than the gauge fields, won't the bubble either collapse or expand on a time scale set by the rate of the Higgs field evolution? The answer is, no. If we choose a starting configuration with a broken phase bubble, and we evolve the Higgs fields without allowing the gauge fields to evolve, the bubble does not collapse but remains a critical bubble indefinitely. It is essential that the electroweak phase transition is a radiatively induced phase transition; the state of the gauge field fluctuations, alone, are sufficient to indicate which phase a configuration is in, and the Higgs condensate cannot expand into the symmetric phase, or collapse inside the bubble, without the gauge field fluctuations changing as well. To demonstrate this point, we have performed an $\eta=\infty$ evolution, meaning an evolution in which only the Higgs fields, and not the gauge fields, are allowed to evolve. It is compared to an evolution in which both evolve, but the gauge fields evolve much more slowly, in Fig. 11. Naturally, the value of $\phi_{\mathrm{av}}^{2}$ changes during each evolution; but in the evolution with frozen gauge fields, it just bounces around a central value, and the bubble is stable. We should expect, then, that $\left\langle\left|\Delta \phi_{\mathrm{av}}^{2} / \Delta t\right|\right\rangle$ and $\mathbf{d}$ will depend on $\eta$. But, as we must check, the product should have a finite limit. We would also like to know how the product $\left\langle\left|\Delta \phi_{\text {av }}^{2} / \Delta t\right|\right\rangle \mathbf{d}$ depends on $\delta m^{2}$; we expect that the dependence is weak.

To check this we have used two values of $\eta, \eta=5$ and $\eta=10$, and measured $\mathbf{d}$ and $\left\langle\left|\Delta \phi_{\mathrm{av}}^{2} / \Delta t\right|\right\rangle$ for each (on a $124^{3}$ lattice at $\delta m^{2}=-0.0082 g^{4} T^{2}$ ). The results are presented in Table III, which shows that the product $\mathbf{d}\left\langle\left|\Delta \phi_{\mathrm{av}}^{2} / \Delta t\right|\right\rangle$ is independent of $\eta$, within numerical errors. We have also re-analyzed the same set of trajectories, sampling $\phi_{\mathrm{av}}^{2}$ half as often; we find as expected that $\mathbf{d}$ and $\left\langle\left|\Delta \phi_{\mathrm{av}}^{2} / \Delta t\right|\right\rangle$ each depend strongly on the sampling rate, but the product does not.

We have also varied the degree of supercooling, to confirm that the dependence of the dynamical prefactors is not very strong. In the $120^{3}$ box we have results at 3 different values of supercooling: $\delta m^{2} /\left(g^{4} T^{2}\right)=-0.0082,-0.0093$ and -0.0104 . These correspond to significantly different bubble probabilities, as shown in Table $\mathbb{I V}$ : the largest supercooling corresponds to bubbles which are $\approx e^{39}$ times more likely to nucleate than with the smallest supercooling. The bubble probability density, Eq. (2.7), is readily evaluated by integrating the data shown in Fig. \&. However, as expected, the dynamical factors are seen to be fairly stable throughout this region, varying only by approximately a factor of two or three, which is in practice insignificant when compared with the differences in probability.

Note the units on $\left\langle\left|\Delta \phi_{\mathrm{av}}^{2} / \Delta t\right|\right\rangle$. The units on the first term in Eq. (2.6) are the same as $1 / \phi_{\mathrm{av}}^{2} \sim 1 / \alpha_{w} T^{2}$; while to get a rate per unit volume we must divide by the volume, $1 / V \sim \alpha_{w}^{3} T^{3}$. Hence the parametric appearance of the nucleation rate is $\propto \alpha_{w}^{5} T^{4} \log (1 / g)$, using $\sigma_{\mathrm{el}} \sim T / \log (1 / g)$. This arises simply from the relation between dimensionless lattice quantities and physical quantities. 


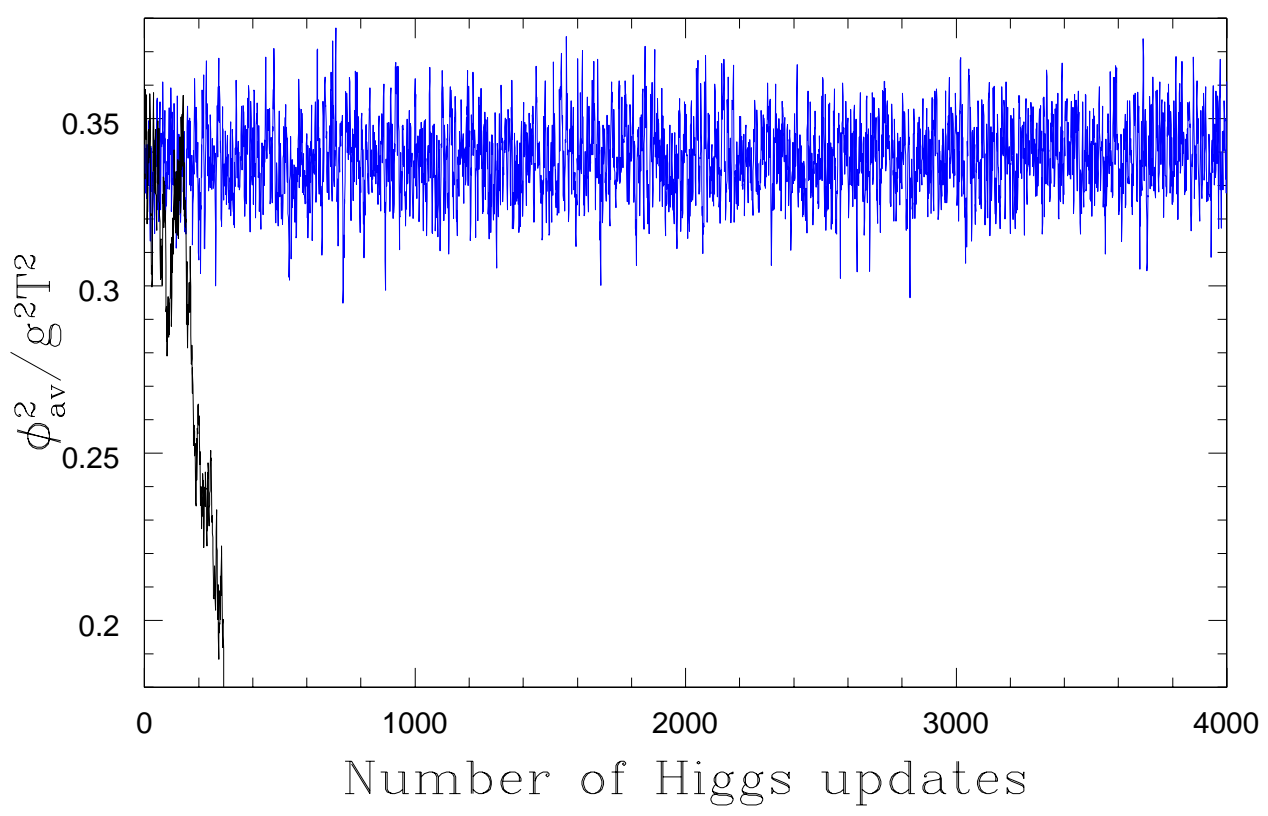

FIG. 11. Two time histories in the $124^{3}$ volume, both starting at the critical bubble: that in which the gauge field is not updated (blue, stays nearly constant) and that in which the gauge field is updated (black). When both fields are updated, the critical bubble can grow, or, in this case, collapse; if only the Higgs fields are updated it does not evolve.

\begin{tabular}{|c|c|c|c|c|}
\hline$\eta$ & $\frac{4 \Delta t}{\sigma_{\mathrm{el}} a^{2}}$ & $\left\langle\left|\frac{\Delta \phi_{\mathrm{av}}^{2}}{\Delta t}\right|\right\rangle \frac{\sigma_{\mathrm{el}}}{\alpha_{w}^{3} T^{4}}$ & $\mathbf{d}$ & $\left\langle\left\langle\frac{\Delta \phi_{\mathrm{av}}^{2}}{\Delta t} \mid\right\rangle \mathbf{d} \frac{\sigma_{\mathrm{el}}}{\alpha_{w}^{3} T^{4}}\right.$ \\
\hline 5 & 1 & $366 \pm 8$ & $0.0169 \pm 0.0028$ & $6.2 \pm 1.0$ \\
\hline 5 & 2 & $240 \pm 7$ & $0.0264 \pm 0.0048$ & $6.3 \pm 1.2$ \\
\hline 10 & 1 & $500 \pm 10$ & $0.0146 \pm 0.0037$ & $7.3 \pm 1.8$ \\
\hline 10 & 2 & $322 \pm 6$ & $0.0218 \pm 0.0048$ & $7.0 \pm 1.6$ \\
\hline
\end{tabular}

TABLE III. Dynamical information $\left\langle\left|\Delta \phi_{\mathrm{av}}^{2} / \Delta t\right|\right\rangle$ and $\mathbf{d}$, varying $\eta$ and sampling each data series at two rates (all on a $124^{3}$ lattice at $\delta m^{2}=-0.0082 g^{4} T^{2}$ ). Each pair of data at fixed $\eta$ comes from the same set of trajectories; comparison shows that $\mathbf{d}$ and $\left\langle\left|\Delta \phi_{\mathrm{av}}^{2} / \Delta t\right|\right\rangle$ depend strongly on sampling frequency, but the product does not. The (fully independent) data sets with different $\eta$ agree within error for $\left\langle\left|\Delta \phi_{\mathrm{av}}^{2} / \Delta t\right|\right\rangle \mathbf{d}$, showing this quantity has good large $\eta$ behavior.

\begin{tabular}{|c|c|c|c|c|}
\hline$\frac{\delta m^{2}}{g^{4} T^{2}}$ & $-\log \left[P_{C} \times \alpha_{w} T^{2}\right]$ & $\left\langle\left|\frac{\Delta \phi_{\mathrm{av}}^{2}}{\Delta t}\right|\right\rangle \frac{\sigma_{\mathrm{el}}}{\alpha_{w}^{3} T^{4}}$ & $\mathbf{d}$ & $-\log \left[\frac{\text { rate }}{V} \frac{\sigma_{\mathrm{el}} / T}{\alpha_{w}^{5} T^{4}}\right]$ \\
\hline-0.00835 & $94.1 \pm 0.6$ & $343 \pm 6$ & $0.025 \pm 0.008$ & $97.0 \pm 0.7$ \\
\hline-0.00951 & $71.0 \pm 0.5$ & $331 \pm 9$ & $0.020 \pm 0.005$ & $74.1 \pm 0.6$ \\
\hline-0.01057 & $54.8 \pm 0.6$ & $314 \pm 8$ & $0.009 \pm 0.004$ & $58.8 \pm 0.7$ \\
\hline
\end{tabular}

TABLE IV. The nucleation rate calculated with 3 different supercoolings, in a $120^{3}, g^{2} T a=4 / 9$ lattice. $P_{C}$ is the probability density that $\phi_{\mathrm{av}}^{2}=\phi_{\mathrm{av}, \mathrm{C}}^{2}$, calculated from Eq. (2.7). The nucleation rate in the fifth column is $P_{C}\left\langle\left|\Delta \phi_{\mathrm{av}}^{2} / \Delta t\right|\right\rangle \mathbf{d} / 2$. 


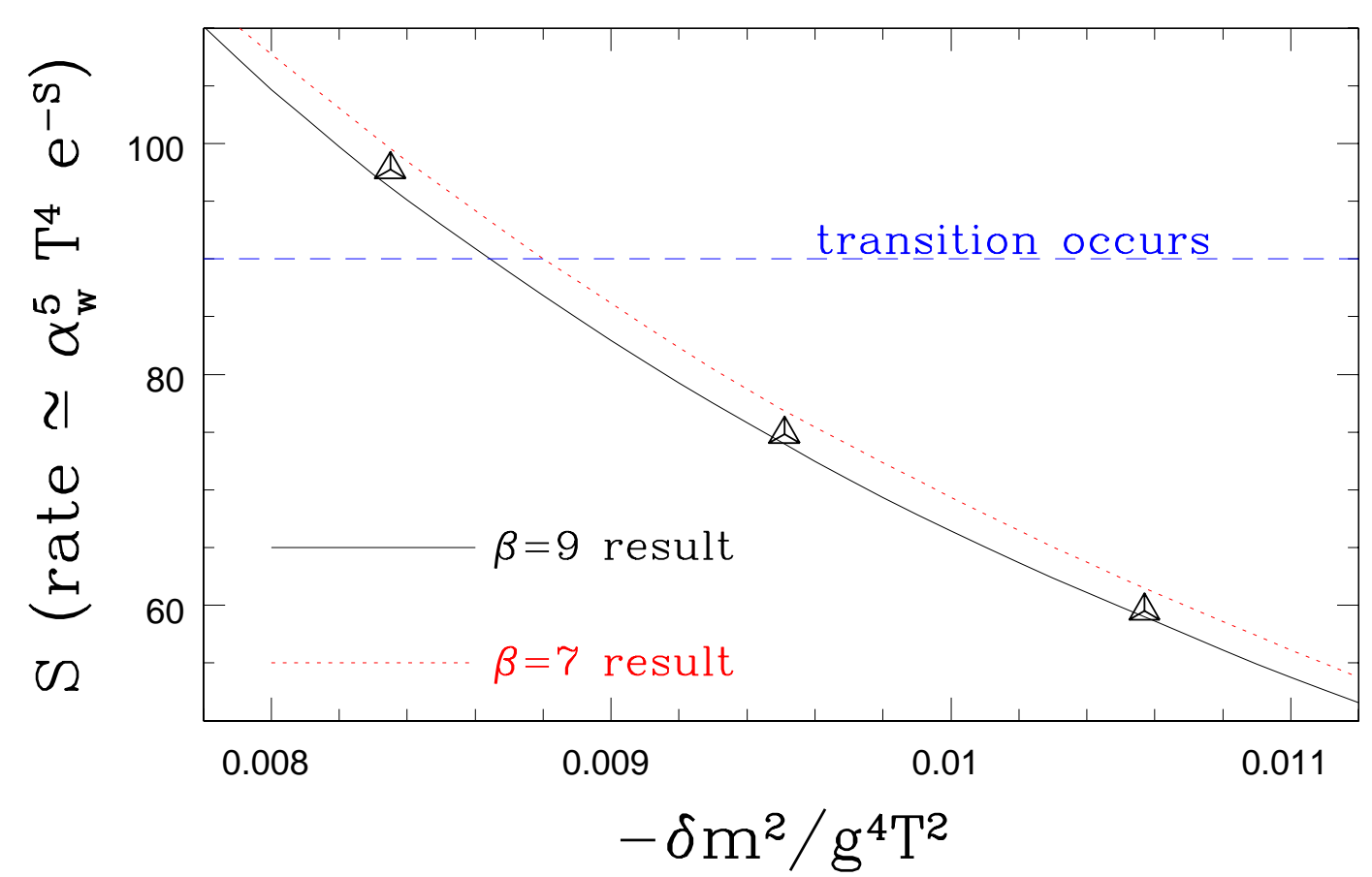

FIG. 12. $S$, as defined in the text, as a function of $\delta m^{2}$, the degree of supercooling, for the $g^{2} T a=4 / 7$ (dotted) and $g^{2} T a=4 / 9,124^{3}$ lattice (solid) data, each using $\left\langle\left|\Delta \phi_{\mathrm{av}}^{2} / \Delta t\right|\right\rangle \mathbf{d}$ as evaluated at $\delta m^{2}=-0.0082 g^{4} T^{2}$. The points are the $120^{3}$ data, using the three evaluations of $\left\langle\left|\Delta \phi_{\mathrm{av}}^{2} / \Delta t\right|\right\rangle \mathbf{d}$ at the values of $\delta m^{2}$ where they were evaluated. Error bars, not shown, are dominated by the error in the determined probability distribution, and are about \pm 1 in $S$.

\section{F. Numerical results and relation to cosmology}

The previous two subsections contain all the ingredients needed to determine the real time rate for bubble nucleation. It remains, first, to put the ingredients together, and second, to determine what value for the nucleation rate is interesting cosmologically. We write the bubble nucleation rate as

$$
\frac{\text { rate }}{V}=\left(\frac{g^{2} T^{2}}{m_{D}^{2}}\right) \log (1 / g) \alpha_{w}^{5} T^{4} \exp (-S) .
$$

For the realistic standard model values $m_{D}^{2}=(11 / 6) g^{2} T^{2}$ and $\alpha_{w} \simeq 1 / 30$, minus the $\log$ of the term in front (evaluating $\log (1 / g)$ using Eq. (3.9)) is 16 , so the rate is $\exp (-(S+16)) T^{4}$. Our result for $S$ is shown, as a function of $\delta m^{2}$, in Figure 12. This represents our final numerical result. It is unfortunate that the numerical effort is too large to perform the calculation for several couplings, and we have also not considered a realistic set of parameters in the MSSM.

What are the errors of $S$ in Figure 12]? Using the numbers in Table IV, for example, we see that the final statistical errors are strongly dominated by the errors of the probability distribution $P$. In addition, there is the systematical error of the real-time update evolution (see sect. IVE). Both of these error sources are easily included by a \pm 2 error band around $S$ in Figure 12 .

We want to know at what value of $S$ the phase transition occurs, so we can determine $\delta m^{2}$ and therefore the amount of supercooling. The relevant picture is discussed in [50. The 
bubbles of broken phase which convert most of the volume into the broken phase, nucleate over a characteristic period of time $t_{\text {nuc }}$, and with a mean separation $d_{\text {nuc }}$, which is also the diameter they grow to. For one such bubble to nucleate per $d_{\text {nuc }}^{3}$ volume in time $t_{\text {nuc }}$, the nucleation rate must be $\sim 1 /\left(t_{\text {nuc }} d_{\text {nuc }}^{3}\right)$. The time scale is set by how fast the nucleation rate is changing; $t_{\text {nuc }} \simeq(d S / d t)^{-1} \simeq(H(T) T d S / d T)^{-1}$, with $H(T)$ the Hubble's constant at temperature $T$. The separation is $d_{\text {nuc }} \sim v_{\mathrm{s}} t_{\mathrm{nuc}}$, with $v_{\mathrm{s}}$ the sound speed $\sim 1$; this is because a bubble is preceded by a shock front propagating at approximately $v_{\mathrm{s}}$, which heats the plasma, and nucleations are suppressed in the heated region. Hence the nucleations take place when

$$
\text { rate } \simeq\left(H \frac{T d S}{d T}\right)^{-4}
$$

From Fig. 12, and from the mass-temperature relation, Eq. (3.3), we see $T d S / d T \sim 2 \times$ $10^{4}$; while from the Friedman equation, $H=\sqrt{4 \pi^{3} g_{*} / 45}\left(T^{2} / m_{\mathrm{pl}}\right) \sim e^{-36.5} T$. Hence the interesting value of $S$ is $16+S \simeq 106$, or $S \simeq 90$. The $g^{2} T a=4 / 7$ data show this value is obtained at $\delta m^{2}=(-0.00880 \pm 0.00010) g^{4} T^{2}$, while the finer lattice data give $(-0.00864 \pm 0.00010) g^{4} T^{2}$. An extrapolation to zero lattice spacing, assuming $O\left(a^{2}\right)$ errors, gives $\delta m^{2}=-0.00840 \pm 0.00020$. Again, this is for $\lambda / g^{2}=0.036$; naturally the amount of supercooling is strongly dependent on the strength of the phase transition, and hence on $\lambda / g^{2}$. This is our final numerical result.

\section{OTHER APPROACHES}

Since we have only computed the nucleation rate nonperturbatively for a single set of parameters, the main application of this work is as a benchmark for studying the performance of other, less first principles means of determining the nucleation temperature, which have previously been used in the literature. For this purpose, we will apply a few of these techniques to the current set of parameters, to see how accurately they determine $\delta m^{2}$. (This actually gives an optimistic appraisal; the dependence of $S$ on $\delta m^{2}$ is strong, so the relative error in $S$ the $\log$ of the nucleation rate is typically almost twice the relative error in $\delta m^{2}$.)

\section{A. Thin wall approximation estimate}

The idea of the thin wall approximation is to hope or assume that the critical bubble is "thin walled," which technically means we assume 3 things:

1. the bubble radius is much greater than the thickness of the phase interface, so we can treat the interface as an infinitely thin geometric surface;

2. the surface tension of the interface, at the nucleation temperature, is the same as at the equilibrium temperature; 
3. the free energy difference between the phases is given by the leading order expression $\Delta V=l \Delta T / T$, with $l$ the latent heat at equilibrium. This neglects the change in $l$ between the nucleation and equilibrium temperatures.

In the limit of small supercooling, all three approximations become exact. Hence the thin wall approximation is appropriate if we are interested in very small $\delta m^{2}$, where the critical bubble free energy is huge. The approximation is in expecting it to continue to work down to where the critical bubble free energy is $S \sim 90 T$.

The thin wall approximation is that a bubble of radius $r$ will have energy

$$
E(r)=4 \pi r^{2} \sigma-\frac{4 \pi}{3} r^{3} \Delta V
$$

with $\sigma$ the equilibrium surface tension and

$$
\Delta V=\frac{l_{\mathrm{eq}} \Delta T}{T}=\frac{-\delta m^{2}}{2} \Delta \phi_{\mathrm{av}}^{2}\left(T_{\mathrm{eq}}\right) .
$$

We find the extremum over all $r$,

$$
\frac{\partial E}{\partial r}=0 \Rightarrow r=\frac{2 \sigma}{\Delta V}, \quad E=\frac{16 \pi \sigma^{3}}{3(\Delta V)^{2}} .
$$

We now estimate that the nucleation rate will be

$$
\text { rate }=\exp (-E / T)\left(\frac{g^{2} T^{2}}{m_{D}^{2}}\right) \alpha_{w}^{5} \log (1 / g) T^{4},
$$

where the technique really provides us no way of getting the non-exponential term we write, but we guess that this is correct on parametric grounds.

For comparison with the case we have studied numerically, we solve for the value of $\delta m^{2}$ required to make $E / T=S=90$, using the nonperturbatively determined values of $\sigma$ and $\Delta \phi_{\mathrm{av}}^{2}\left(T_{\mathrm{eq}}\right)$ from the last section, $\sigma=0.075 g^{4} T^{3}$ and $\Delta \phi_{\mathrm{av}}^{2}\left(T_{\mathrm{eq}}\right)=2.53 g^{2} T^{2}$. Substituting into

$$
-\delta m^{2}=\left(\frac{64 \pi \sigma^{3}}{3\left(\Delta \phi_{\mathrm{av}}^{2}\left(T_{\mathrm{eq}}\right)\right)^{2} E}\right)^{1 / 2}
$$

gives $\delta m^{2}=-0.0070 g^{4} T^{2}$. This is low by about $20 \%$ from the nonperturbative value.

Alternately, we could ask how accurately the thin wall approximation predicts the nucleation rate at the actual value of $\delta m^{2}$. Plugging in the value of $\delta m^{2}$ found in the last section gives $S=62$, which is off by almost a third.

It is clear that the thin wall approximation is doomed to errors of this magnitude when applied where $E / T \sim 90$. Note that the radius of the critical bubble, for $\delta m^{2}$ determined above, is

$$
r=\left(\frac{3 E}{4 \pi \sigma}\right)^{1 / 2}
$$

But it is impossible for the thickness of the interface to be less than $\sim \sqrt{T / \sigma}$; even if the intrinsic thickness were somehow thinner, the surface fluctuations would generate such 
a thickness. Hence $r$ never greatly exceeds the surface thickness if we are interested in $E / T \sim 90$. However, all considering, the thin wall approximation (given nonperturbative inputs) does pretty well.

We should comment that, although the thin wall approximation requires nonperturbative inputs, it is much easier to determine $\Delta \phi_{\mathrm{av}}^{2}$ and $\sigma$ on the lattice than to directly compute the nucleation rate. In particular, we could think realistically of doing a "scan" of $\Delta \phi_{\mathrm{av}}^{2}$ and $\sigma$ at several parameter values, in the standard model or one of its extensions, but to do the nucleation rate at numerous values of parameters would probably be beyond what is currently a reasonable amount of numerical effort. Hence the thin wall approximation may be a reasonable approach to getting "rough and ready" nucleation information.

\section{B. Perturbative estimate}

The traditional method for determining the bubble nucleation rate in the context of a perturbative treatment of the strength of the phase transition (see for instance [50 52]) is to approximate the free energy (effective action) to be the tree kinetic term for the (gauge fixed) Higgs condensate $\phi$, plus an effective potential term computed at some order in the loop expansion,

$$
E=\int d^{3} x\left(\frac{1}{2}(\nabla \phi)^{2}+V(\phi)\right)
$$

It is also possible to consider radiative corrections to the Higgs field kinetic energy, as we discuss in the next subsection.⿴囗十

We begin with the simplest possible estimate. We take the one loop effective potential, Eq. (3.8), reproduced here for convenience:

$$
V_{1 \text { loop }}=\frac{m_{H T}^{2}}{2} \phi^{2}-\frac{g^{3}}{16 \pi} \phi^{3} T+\frac{\lambda}{4} \phi^{4} .
$$

Here we have neglected scalar loops, which give a contribution down relative to the $\phi^{3}$ term by $\left(\lambda / g^{2}\right)^{3 / 2}$. We can see from Eq. (5.8) that at

$$
m_{H T}^{2}=m_{T_{\mathrm{eq}}}^{2}(1 \text { loop })=\frac{g^{6} T^{2}}{128 \pi^{2} \lambda}
$$

\footnotetext{
${ }^{11}$ We note that a full-fledged analytical computation of the nucleation rate in the spirit of the Langer method is very difficult in radiatively induced first order transitions. The problem is how to distinguish the fluctuations which give you the effective potential from the fluctuations of the bubble in this potential. The results from a cubic anisotropy model (a simple spin model) calculation by Strumia and Tetradis [53] display a dramatic dependence on how the separation of the fluctuations is done; since the physics cannot depend on the separation of fluctuations, this effect must be an artifact of the approximations done in the calculation. On the other hand, if there is a sufficiently strong first order transition already at tree level, the Langer method is relatively straightforward to apply reliably 54 56.
} 
there are two degenerate minima with

$$
\phi=0 \quad \text { and } \quad \phi=\phi_{0}(1 \mathrm{loop})=\frac{g^{3} T}{8 \pi \lambda} .
$$

At best perturbation theory is an expansion in $\sim\left(g^{2} T / m_{W}\right) \sim g T / \phi$, hence in $\lambda / g^{2}$. Two loop corrections will give a correction to $\phi_{0}$ of order $\left(\lambda / g^{2}\right) \phi_{0}$, which is larger than the scalar loop contribution we neglected above. This justifies neglect of the scalar loop. एా

One then assumes, reasonably, that the critical bubble, the saddle point of Eq. (5.7), will have spherical symmetry, and looks for the saddle point of

$$
E=4 \pi \int_{0}^{\infty} r^{2}\left(\frac{1}{2}\left(\partial_{r} \phi(r)\right)^{2}+V(r(\phi))\right)
$$

over all $\phi(r)$, with the boundary condition that $\phi(r)$ go to zero at large $r$, so we have a bubble in the symmetric phase. One finds the saddle point free energy as a function of $m_{H T}^{2}$ and looks for where it takes on the desired value, say $E / T=S=90$. The difference from the equilibrium $m_{H T}^{2}$ is the degree of supercooling we are after. (As in the previous section we will simply assume that the bubble zero modes etc. give a dimensionful prefactor of $\sim \alpha_{w}^{5} T^{4}$. Since even a change in the numerical value of this prefactor by a factor of 1000 would represent only a change of 7 in the log of the nucleation rate, our results depend only weakly on this treatment.)

We are not aware of a purely analytic way to find the saddle point of Eq. (5.11), but the well known overshoot-undershoot algorithm is both efficient and accurate. We find, for $\lambda / g^{2}=0.036$, that

$$
\delta m^{2}=-0.0058 g^{4} T^{2}, \quad \sigma_{\text {equilibrium }}=\int_{0}^{\phi_{0}} \sqrt{2 V} d \phi=\frac{g^{9} T^{3}}{3072 \sqrt{2} \pi^{3} \lambda^{5 / 2}}=0.0302 g^{4} T^{3}
$$

In comparison to the nonperturbative values these are both fairly far off. The degree of supercooling is $2 / 3$ of the right value and the surface tension is $40 \%$ of the nonperturbative value. One loop perturbation theory is NOT accurate even at $\lambda / g^{2}=0.036$, though it is not completely wrong.

\footnotetext{
${ }^{12} \mathrm{As}$ an aside we mention that the expressions usually written for scalar loops (refs. 57,58, though not ref. [59]) must be inconsistent because they depend on $m_{H T}^{2}$ in a way which does not satisfy Eq. 2.5. The problem is that they were derived using a Higgs mass in loops which does not correspond to the curvature of the potential; the value of $m_{H}^{2}$ used in loops includes the second derivative of the $\lambda \phi^{4}$ potential term but not of the negative cubic term, which is of the same size in the interesting region and cannot be neglected in any consistent approximation scheme. If we include terms in the effective potential in the spirit of an expansion in $\lambda / g^{2}$ then no reference to the scalar self-coupling or $m_{H T}^{2}$ appears in any loop induced term until $O\left(\left(\lambda / g^{2}\right)^{3 / 2}\right)$, when an infinite class of diagrams must be resummed, corresponding to a single Higgs loop but including iterated one loop mass corrections from gauge boson loops within the 3D theory (not just the nonzero Matsubara frequency loops).
} 


\begin{tabular}{|c|c|c|c|}
\hline Potential used & Wave Function $Z$ & $-\delta m^{2} / g^{4} T^{2}$ & $\sigma / g^{4} T^{3}$ \\
\hline 1 Loop & Tree & 0.0058 & 0.0302 \\
\hline \multirow{3}{*}{ 2 Loop } & Tree & 0.0168 & 0.088 \\
& $Z_{\text {exp }}$ & 0.0109 & 0.072 \\
& $Z_{\text {pade }}$ & 0.0115 & 0.074 \\
\hline \multirow{3}{*}{ "nonperturbative" } & Tree & 0.0151 & 0.097 \\
& $Z_{\text {exp }}$ & 0.0110 & 0.083 \\
& $Z_{\text {pade }}$ & 0.0114 & 0.084 \\
\hline \hline Nonpert. Result & & 0.0084 & 0.075 \\
\hline
\end{tabular}

TABLE V. Supercooling and surface tension, using several analytical or semi-analytical approaches, compared with the full nonperturbative answer (last line). The meanings of $Z_{\text {pade }}$ and $Z_{\text {exp }}$ are explained in the next subsection. The one loop result gives too small results; all other results give too large an answer.

We therefore go on to the two loop effective potential. Neglecting powers of $\lambda$ and setting $m_{H T} \ll m_{W}$ to zero within loops, the new term in the effective potential is 60]

$$
V_{2 \text { loop }}=V_{1 \text { loop }}-\frac{51}{512 \pi^{2}} g^{4} \phi^{2} T^{2} \log (\phi / g T),
$$

where the choice of $g T$ inside the logarithm is a convenient choice of renormalization point; a different choice can be absorbed into a shift in $m_{H T}^{2}$. The justification for neglecting scalar effects is the same as before; for more discussion see footnote 12.

Including this term in the effective potential, we find that, while $\phi_{0}^{2}=2.19$ is still smaller than the nonperturbative $\Delta \phi_{\mathrm{av}}^{2}$, we now get too large a surface tension and too much supercooling, by a substantial margin:

$$
\sigma_{2 \text { loops }}=0.088 g^{4} T^{3}, \quad \delta m^{2}(2 \text { loops })=-0.0168 g^{4} T^{2} .
$$

The amount of supercooling is too large by a factor of 2 . At the value of $\delta m^{2}$ found nonperturbatively, $S_{2}$ loop $>260$ is almost 3 times too large. All the results for various effective potentials are summarized in Table $\square$.

It is also possible to define a nonperturbative "effective potential for $\phi$," as follows. We first find the largest volume for which the configurations with $\phi_{\mathrm{av}}^{2}$ intermediate between the pure phase values, do not show phase segregation. This turns out to be a surprisingly large volume. To see this, recall why, in a larger box, a configuration with $\phi_{\mathrm{av}}^{2}$ intermediate between homogeneous phase values is a mixed phase configuration, rather than an extensive region with the intermediate field value. The effective potential we will eventually derive is shown in Fig. 13. The state half way between minima has negative curvature. Infrared Higgs field fluctuations are therefore spinodally unstable. If we force $\phi_{\mathrm{av}}^{2}$ to maintain its value, this prevents the zero mode from growing, ie. it keeps $\phi$ from shifting value homogeneously through the box; but small $k$ modes will be unstable. Any mode with $k<\omega_{-}$, with $\omega_{-}=\sqrt{-V^{\prime \prime}}$ evaluated at the unstable point in question, will be unstable to grow. However, in a finite volume, there is a discrete spectrum of $k$ modes. The lowest nontrivial mode has $k=2 \pi / L ;$ so in any volume smaller than $L=2 \pi / \omega_{-}$, configurations with intermediate $\phi_{\mathrm{av}}^{2}$ 

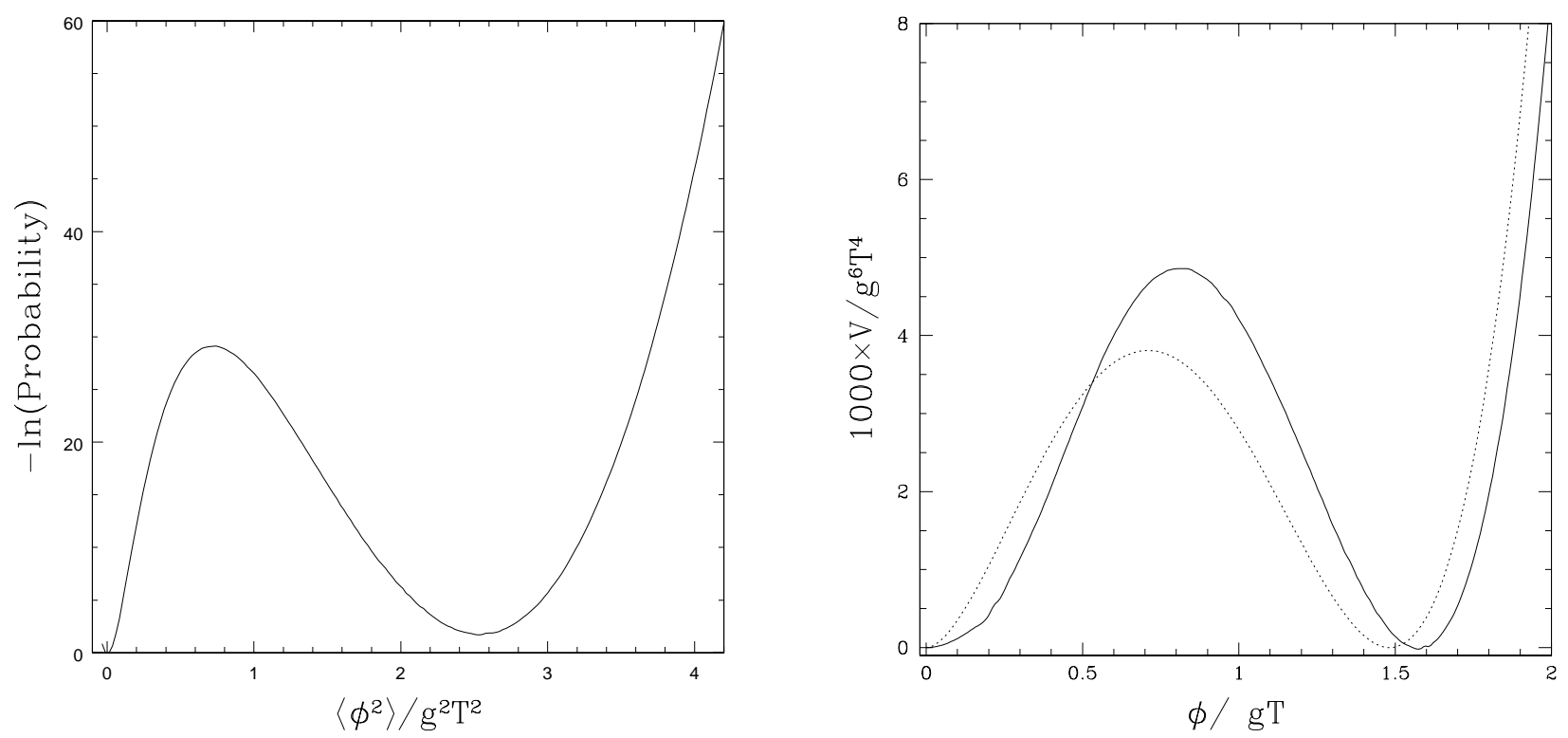

FIG. 13. Left: free energy $\left(-\ln\right.$ (probability)) distribution as a function of $\phi_{\text {av }}^{2}$ in a $40^{3}$ box at $a=4 / 9 g^{2} T(\beta=9)$. The numerical errors are $<0.2$ and not shown on the plot. Right: plot at left interpreted as an effective potential for $\phi$, as described in the text. The dotted line is the 2 loop perturbative result, included for comparison.

will be homogeneous. In larger boxes they will be inhomogeneous, containing a region closer to one phase and a region closer to the other.

Now, we determine $F\left(\phi_{\mathrm{av}}^{2}\right)$, meaning $-\log \left(\operatorname{Prob}\left(\phi_{\mathrm{av}}^{2}\right)\right)$, in such a "large but not very large" volume. In practice we use a $40^{3}$ lattice at $\beta=9$, meaning a volume $17.8 / \mathrm{g}^{2} \mathrm{~T}$ on a side. This is pretty big, though the volumes we used to study critical bubbles were typically 3 times longer on a side. We then write $V=F / V$ and $\phi=\sqrt{\phi_{\mathrm{av}}^{2}-\phi_{\mathrm{av}}^{2} \text { (s phase) }}$, with $\phi_{\mathrm{av}}^{2}$ (s phase) the lower local minimum of $F\left(\phi_{\mathrm{av}}^{2}\right)$ at $T_{\mathrm{eq}}$. That is, we throw away the part of the picture on the left in Fig. 13 which lies at smaller $\phi_{\mathrm{av}}^{2}$ than the first minimum, and rescale the $x$ axis for the rest. We take the result to be a "nonperturbative measured $V(\phi)$ ", shown at the right in the figure.

The advantages of getting an effective potential in this way are that it should automatically get the right latent heat, and will show the disappearance of the phase transition at large $\lambda / g^{2}$. The disadvantages are that it is numerically expensive (though less so than a direct determination of the nucleation rate), is somewhat arbitrary (how exactly do we choose a volume, for instance?), and is still only approximative. In particular, it is not at all clear that it is reasonable to assume that $\phi$ as defined above will have canonically normalized gradient energies.

The "nonperturbative effective potential" has a larger separation between minima, but the height of the barrier rises relative to the two loop potential more weakly than as $\phi_{0}^{4}$. As a result it gives a slightly lower degree of supercooling, $\delta m^{2}=-0.0151$, as summarized in Table $\nabla$. Note however that the surface tension is further off than in two loop perturbation theory. What this exercise teaches us is that the difference between the perturbative and the nonperturbative values of $\sigma$ and $\delta m^{2}$ are not primarily because of the limitations of the 2 loop effective potential. 

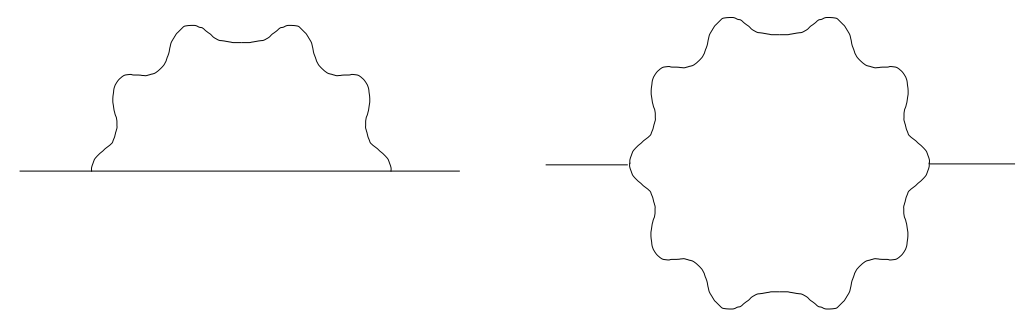

FIG. 14. Diagrams which lead to $O\left(\lambda / g^{2}\right)$ important Higgs wave function corrections. The vertices in the diagram at right only exist because there is a condensate.

\section{Perturbative estimates including wave function corrections}

We have just found that estimates of the degree of supercooling and of the surface tension were not substantially improved by passing from the one loop to the two loop effective potential, using the tree level Higgs field kinetic term in each case. In fact this result is not surprising. While the two loop effective potential can tell us the latent heat $l \propto \phi_{\text {av }}^{2}$ at next to leading order in $\lambda / g^{2}$, the procedure we used is still correct only at leading order in $\lambda / g^{2}$ for determining the quantities we want, because it does not incorporate thermal corrections to the Higgs field wave function. In this subsection we see what happens when we incorporate Higgs wave function corrections which make the calculation complete to next to leading order in $\lambda / g^{2}$.

Using the one loop effective potential, we can get the parametric estimate $\phi_{0} \sim g^{3} T / \lambda$, so $m_{W}(\phi) \sim g^{4} T / \lambda$ in the broken phase and inside the wall. The wall thickness, on the other hand, is set by the Higgs boson mass in either phase, $L_{\text {wall }} \sim 1 / m_{H T} \sim g^{3} T / \sqrt{\lambda}$. Therefore, up to a correction suppressed by a half power of $\lambda / g^{2}$, we may take the $W$ bosons to be heavy compared with the reciprocal wall width, and treat the Higgs condensate as a homogeneous background for them. For this reason it is possible to incorporate the leading non-potential correction as a wave function correction for the Higgs field,

$$
\frac{1}{2}(\nabla \phi)^{2} \quad \Rightarrow \quad \frac{Z(\phi)}{2}(\nabla \phi)^{2}
$$

Expanding the diagrams shown in Fig. 14 to second order in external momenta (treated as much less than $m_{W}$ ), we find a correction to the Higgs gradient term which is, in Landau gauge,

$$
Z(\phi)=1+\delta Z(\phi)=1-\frac{11}{32 \pi} \frac{g^{2} T}{m_{W}}=1-\frac{11}{16 \pi} \frac{g T}{\phi},
$$

in agreement with the expression found by Bödeker et. al. [61] (see also [62], where these wave function corrections have been considered at length).

Both the corrections from Higgs fields, and from higher order in the $p \ll m_{W}$ expansion, will give at most $O\left(\lambda^{3 / 2} / g^{3}\right)$ corrections to $\sigma$ and $\delta m_{H T}^{2}$; whereas we see from the estimate for $\phi_{0}$ that the above term contributes at $O\left(\lambda / g^{2}\right)$. It will drive down both $\sigma$ and $\delta m^{2}$, since it lowers the gradient energy contribution to the energy, and so makes it cheaper to have bubbles or interfaces. We should mention that to compute the $\left(\lambda / g^{2}\right)^{3 / 2}$ corrections, we 
would have to perform a fluctuation determinant, since the Higgs field mass and the width of the interface are parametrically the same.[3]

Unfortunately $Z(\phi)$ goes to zero at a finite value of $\phi$. At the value of $\phi$ where this happens, the condensate is so weak that perturbation theory is also breaking down; the failure signals that neglected higher order effects become essential. To deal with this, we will make an Ansatz for those effects, chosen to maintain the correct large $\phi$ behavior of $Z(\phi)$ but to prevent $Z(\phi)$ from going to zero. We have considered two choices; to approximate

$$
Z_{\text {exp }}=\exp (-\delta Z), \quad \text { and } \quad Z_{\text {pade }}=\frac{1}{1-\delta Z} .
$$

Since we do not know the higher order behavior of $Z$, we do not know which of these is more sensible. If the answers we get depend strongly on which one we take, that is an indication that the perturbative expansion is failing and we cannot trust either.

When we re-compute $\delta m^{2}$ and $\sigma$, including these wave function corrections, we get a result which is much closer to the nonperturbative value, see Table $\nabla$. Further, the choice of how to resum higher terms in $Z(\phi)$ appears not to matter much. However, the supercooling is still over-estimated by about $25 \%$. At $\delta m^{2}$ where the nonperturbative calculation shows the phase transition takes place, where $S_{\text {nonpert }}=90$, the 2 loop potential with Padé resummed self-energy corrections gives $S=143$, more than $50 \%$ high.

In summary, using a perturbatively computed effective potential but tree Higgs kinetic terms appears to work badly. Higgs wave function corrections are important and improve the performance of the perturbative treatment; they should be included in any subsequent work which tries to study electroweak bubble nucleation by perturbative means. However, even with wave function corrections, perturbation theory is still not a very accurate way to treat bubble nucleation.

\section{CONCLUSIONS}

In this paper we have presented a technique for determining bubble nucleation rates in theories with classical infrared thermodynamics and dynamics, in a fully nonperturbative way-even where the rate is exponentially small. The technique can be considered a generalization of Langer's formalism [28], which replaces the approximate saddle point expansion of that method with a nonperturbative Monte Carlo calculation, and takes care to treat correctly the microscopic dynamics of the nucleation process. The procedure uses an interesting mixture of multicanonical Monte Carlo and real time techniques. Within the context of the dimensional reduction approximation for the thermodynamics, and Bödeker's

\footnotetext{
${ }^{13}$ Such a fluctuation determinant calculation has been performed by Baacke [63], and has also recently been considered by Parnachev and Yaffe [64]; but since neither reference uses the two loop effective potential, their results cannot be considered correct through to $O\left(\lambda / g^{2}\right)$. It is not clear to us how to simultaneously perform the fluctuation determinant and to incorporate the two loop gauge contributions to the effective potential. Without resolving this question, the calculation of the fluctuation determinant is not justified in the sense of an expansion in $\lambda / g^{2}$.
} 
effective theory for the dynamics [15, our treatment is exact up to small and controllable numerical errors. It is useful when the microscopic physics is known well enough, and suitably amenable to a lattice treatment, to permit an accurate first principles Monte Carlo calculation. In particular, it can be applied at the electroweak phase transition.

We have applied the technique to the electroweak phase transition in the standard model, at a value of the coupling which is just enough to preserve any baryon number after the transition; the ratio of (3-D effective theory couplings) was taken as $\lambda / g^{2}=0.036$. The degree of supercooling is such that the thermal Higgs mass squared falls by $\delta m^{2}=-0.00840 g^{4} T^{2}$ from its equilibrium value.

The main value of this measurement is that we can compare it to the results of more traditional and less first principles calculations. We find that the most common method in the recent literature, using the two loop effective potential but tree level Higgs kinetic term, is very unreliable. For the parameters considered it overestimates the amount of supercooling by a factor of 2 , even though it gets $\phi_{0}$, the length of the Higgs field in the broken phase, to within $10 \%$ error. If one considers the action of the critical bubble, it is even further off. Including Higgs field wave function corrections substantially improves the accuracy; the amount of supercooling is then only overestimated by about $25 \%$. The remaining discrepancy cannot be attributed to the inaccuracy of the effective potential; a nonperturbative "effective potential for $\phi$ " is off by the same amount. On the other hand, a thin wall estimate, with nonperturbative inputs, does quite well-it is off by $20 \%$, in the opposite direction.

It should be straightforward to apply our technique to the more phenomenologically interesting MSSM or NMSSM, which can support viable baryogenesis.

\section{Acknowledgments}

We thank Mikko Laine for many useful discussions. KR acknowledges partial support from EU TMR grant FMRX-CT97-0122. A part of the simulations have been run on the Cray T3E at the Center for Scientific Computing, Espoo, Finland.

\section{APPENDIX A: DISCUSSION: INDEPENDENCE ON CHOICE OF MEASURABLE}

In this appendix we justify why the procedure presented in Section $\mathbb{1}$ of the main text "works," and in particular why the determined rate should be independent of the choice of measurable.

Fix attention to a particular volume, and a particular value of $T<T_{\text {eq }}$, at which the nucleation rate is small. We must begin by clarifying the definition of the nucleation rate. First, we must be able to say whether or not a configuration is in the metastable phase. This requires that we possess some measurable in terms of which the free energy shows a "two well" structure, like Fig. 3, with the probability at $(C)$ exponentially smaller than at $(A)$. For simplicity of notation let us assume that $\phi_{\mathrm{av}}^{2}$ serves this purpose.

We can now draw two lines, roughly at $(B)$ and $(D)$ in Fig. 3; all we require is that they be well between $(C)$ and the local minima, such that the likelihood to be at $(B)$ is exponentially greater than the likelihood to be at $(C)$, but exponentially less likely than to be at $(A)$ (and similarly $(D)$ is exponentially more likely than $(C)$ but less likely than the broken phase). We say that a configuration is "definitely in the metastable phase" if it has 
a value of $\phi_{\mathrm{av}}^{2}$ to the left of $(B)$, and is "definitely in the broken phase" if it has a value to the right of $(D)$.

The intuitive meaning of the nucleation rate is the following. Take the canonical ensemble, and throw out all the configurations to the right of $(B)$, leaving only the ones which are clearly in the metastable minimum. Now carry out the time evolution for a "medium" amount of time, $t_{\text {medium }}$, exponentially longer than any microscopic time scale, but exponentially shorter than the time it takes for most of the metastable configurations to escape to the broken phase. At the end of the time evolution, look to see how much of the ensemble is definitely in the broken phase, ie. to the right of $(D)$. Define that fraction, divided by $V t_{\text {medium }}$, to be the spacetime rate of nucleations.

This intuitive meaning of the nucleation rate will be our definition. Note that it is equivalent to the following. Consider the set of all trajectories of length $t_{\text {medium }}$, starting from any configuration (symmetric, broken, or in between) with appropriate Boltzmann weight. The nucleation rate per unit volume is

$$
\text { rate }=\frac{\mathrm{P}(\text { symm } \rightarrow \text { broken })}{V t_{\text {medium }} \mathrm{P}(\text { symm } \rightarrow \text { symm })},
$$

where $(\mathrm{P})$ means the fraction of the trajectories satisfying the given condition. In other words, find what fraction of trajectories start in the symmetric phase and end in the broken one; and divide by the number which start and end in the symmetric phase, the volume, and the time. (The denominator should read $\mathrm{P}($ symm $\rightarrow$ left of $(D)$ ), but since configurations between $(B)$ and $(D)$ are exponentially rare the difference is exponentially small.) This definition of a rate depends on our exact choices for $(B),(D)$, and $t_{\text {medium }}$, but the sensitivity should be exponentially weak - unless there is some additional long time scale in the problem, besides the nucleation rate, in which case our technique probably fails. We can think of the nucleation problem in terms of sampling over the space of real time trajectories, and the sampling may be taken using the equilibrium ensemble. The goal is now to show that our technique correctly determines the number of crossing trajectories, relative to all trajectories which remain near the symmetric phase; and that this does not depend sensitively on our choice of measurable.

From here on we will consider our technique applied to two classes of dynamics. The first is Hamiltonian dynamics, for a system where the phase space is the tangent bundle over the space of configurations. We further assume that the Hamiltonian is a function of space, plus (a constant times) the inner product function on the tangent space. In other words, the momenta appear quadratically in the Hamiltonian, and in any orthonormal basis for the momenta the quadratic form is a multiple of the identity. Probably these conditions are too strong, and we could work with any Hamiltonian system which could be written as a fiber bundle over a configuration space; but the treatment would be more complicated. The other class of dynamics we consider is Langevin dynamics, where the noise term is Gaussian, white, and additive. White means there are no unequal time correlators in the noise, in which case it is described by a weight function on the tangent space; we take that weight function to be of the same form as the momentum distribution just described for the Hamiltonian case. Probably one could extend our technique to the case where the noise is stronger in some coordinate directions, or has an amplitude which varies in space. However, such Langevin systems are notoriously subtle, see eg. 65, 66. 


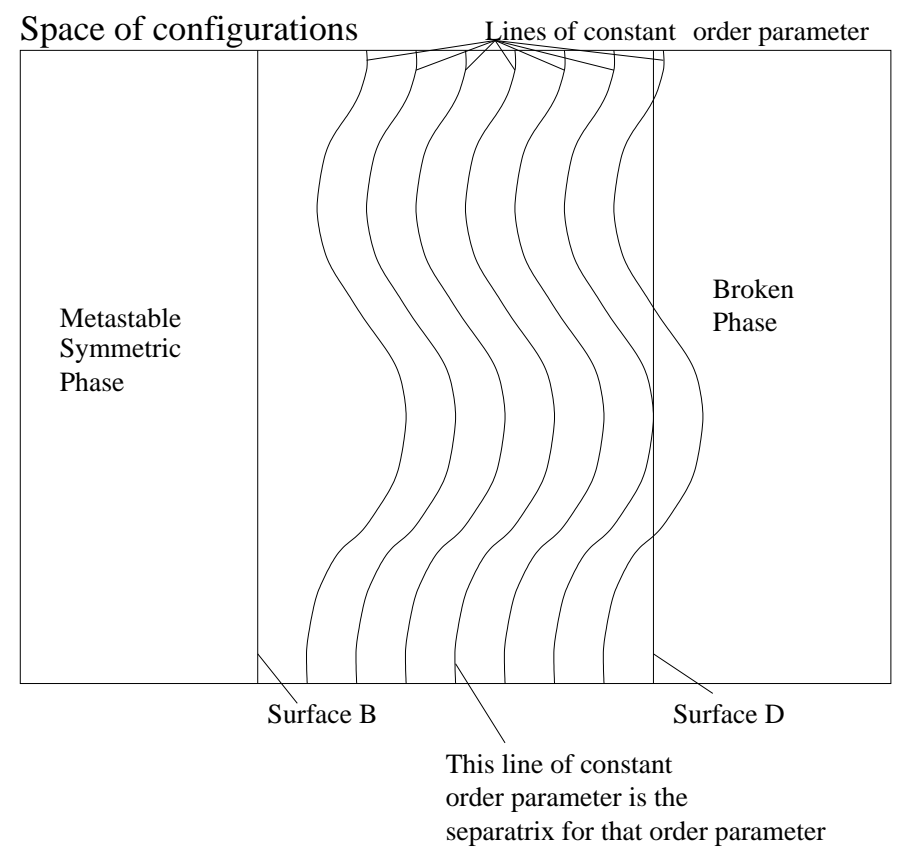

FIG. 15. A cartoon of how configuration space is foliated by a measurable-here the lines of constant measurable do not coincide with the ones which determine the surfaces $B$ and $D$.

Consider two measurables, call them $\mathcal{O}_{1}$ and $\mathcal{O}_{2}$. (They could for instance be $\phi_{\text {av }}^{2}$ and some other volume average of a local observable; or $\mathcal{O}_{2}$ could for instance be the maximum over all centerpoints for spheres of radius $r$, of $\int_{\text {sphere }} 2 \Phi^{\dagger} \Phi$.) For each observable we can make a free energy plot and identify some least likely value of $\mathcal{O}, \mathcal{O}_{\text {crit }}$. The measurable $\mathcal{O}$ is a map from the space of configurations to the real numbers; the subspace which gives a particular value of $\mathcal{O}$ is a codimension 1 surface in the space of configurations. Hence defining a measurable also defines a foliation of the configuration space. We will call the special surface, consisting of all configurations with $\mathcal{O}=\mathcal{O}_{\text {crit }}$, the separatrix for the measurable $\mathcal{O}$. This notation is in keeping with [67], where many of the root ideas of our algorithm can be found. One would like the separatrix to separate the configurations more likely, under time evolution, to go to the symmetric phase, from those more likely to go to the broken phase.

Note that $(B)$ and $(D)$ also give codimension 1 surfaces in the configuration space, namely the surfaces where $\phi_{\mathrm{av}}^{2}$ takes on the values $(B)$ and $(D)$; we will call these surfaces $B$ and $D$. To one side of the surface $B$ is the metastable phase, to the far side of surface $D$ is the broken phase, and in between are intermediate configurations. For the measurable $\mathcal{O}$ to be useful, we require that the $\mathcal{O}$ separatrix carry all but an exponentially small part of its weight between the surfaces $B$ and $D$. We give a cartoon of how these surfaces in configuration space look, in Fig. 15 .

It is possible to define an ideal operator, $\mathcal{O}_{\text {ideal }}$, which will provide an ideal separatrix for distinguishing configurations which are in the domain of attraction of one or the other phase. Namely, we define

$$
\mathcal{O}_{\text {ideal }}(\text { config })=\int_{\text {traj. through config }} \Theta\left(\phi_{\text {av }}^{2}\left(\operatorname{config}\left(t_{\text {medium }}\right)\right)<(B)\right) .
$$

$\mathcal{O}_{\text {ideal }}$ of a configuration is the fraction of trajectories, starting at that configuration, which are in the metastable phase after time $t_{\text {medium}}$. For Hamiltonian dynamics, the measure on 
the space of trajectories through a configuration is just the canonical measure of the tangent space; a point in the tangent space uniquely defines a trajectory. For Langevin dynamics, the measure for the space of trajectories through a point is given by the measure of realizations of the noise, with each trajectory corresponding to the noise realization which generates it. We do not use $\mathcal{O}_{\text {ideal }}$ in our work because its measurement is impractical.

The method presented in the main text for the determination of the nucleation rate, applied to an observable $\mathcal{O}$, can be phrased as follows: first, we find the probability distribution as a function of the value of $\mathcal{O}$; that is, we find the integral along each surface of constant $\mathcal{O}$ of the weight of the canonical ensemble. This gives

$$
\frac{\mathrm{P}\left(\left|\mathcal{O}-\mathcal{O}_{\text {crit }}\right|<\epsilon / 2\right)}{\epsilon \mathrm{P}\left(\mathcal{O}<\mathcal{O}_{\text {crit }}\right)} \propto \int_{\text {surf }} d(\text { surf. area }) \exp (-H / T)[d \mathcal{O} / d(\text { normal })]^{-1},
$$

the integral over the surface of the Boltzmann weight, divided by the surface normal derivative of $\mathcal{O}$. The surface normal derivative appears because, the larger the derivative is, the narrower is the region over which $\mathcal{O}$ differs by less than $\epsilon / 2$ from $\mathcal{O}_{\text {crit }}$.

Multiplying by $\langle|\Delta \mathcal{O} / \Delta t|\rangle$ precisely compensates for this normal derivative factor. This is because the mean value of $|\Delta \mathcal{O} / \Delta t|$, at any point in configuration space, is proportional to the gradient of $\mathcal{O}$ at that point. To see this, note first that only motion normal to the surface of constant $\mathcal{O}$ matters. Next note that, by our requirements on the dynamics, the rms. metric distance traveled in one direction, on averaging over possible momenta (or Langevin noise realizations), is independent of position in configuration space or direction considered. Therefore

$$
\left\langle\left|\frac{\Delta \mathcal{O}}{\Delta t}\right|\right\rangle \propto \frac{\int_{\text {surf }} d(\text { surf. area }) \exp (-H / T)}{\int_{\text {surf }} d(\text { surf. area }) \exp (-H / T)[d \mathcal{O} / d(\text { normal })]^{-1}} .
$$

The product is proportional to the Boltzmann weighted area of the separatrix, and hence the flux through the separatrix, as claimed in the body of the paper.

The flux through the separatrix will clearly differ for different choices of $\mathcal{O}$, as illustrated in Fig. 16. It remains to show that $\mathbf{d}_{\mathcal{O}}$, as defined in Eq. 2.9, precisely turns the correctly normalized flux of trajectories through the separatrix into a count of trajectories which mediate nucleations. Fig. 16 illustrates why this is true. First consider a trajectory which crosses the separatrix an even number of times and returns to the phase it came from, like Trajectory 1 in the figure. If the trajectory crosses $n$ times, it gets counted $n$ times in the sampling procedure, once at each crossing of the separatrix. Each count is with the same weight. For Hamiltonian dynamics this is because the evolution conserves energy (hence the Boltzmann factors are all the same) and phase space measure. For Langevin dynamics it is because the Langevin dynamics correctly generate the thermal ensemble. In either case, the number of times the trajectory gets sampled is in proportion to its contribution to the flux, and each time it contributes zero to $\mathbf{d}$; hence $\mathbf{d}$ correctly accounts for the number of nucleations (zero) the trajectory causes.

Next consider a trajectory which does get from the metastable phase to the stable one. It is guaranteed to cross both separatrices an odd number of times, because each is to the right of $B$ where the trajectory starts, and to the left of $D$ where it ends. If the trajectory crosses a separatrix $n$ times, it will appear in the average, used to determine $\mathbf{d}$, $n$ times, each with the same weight; and each appearance contributes $1 / n$ to the determination of 


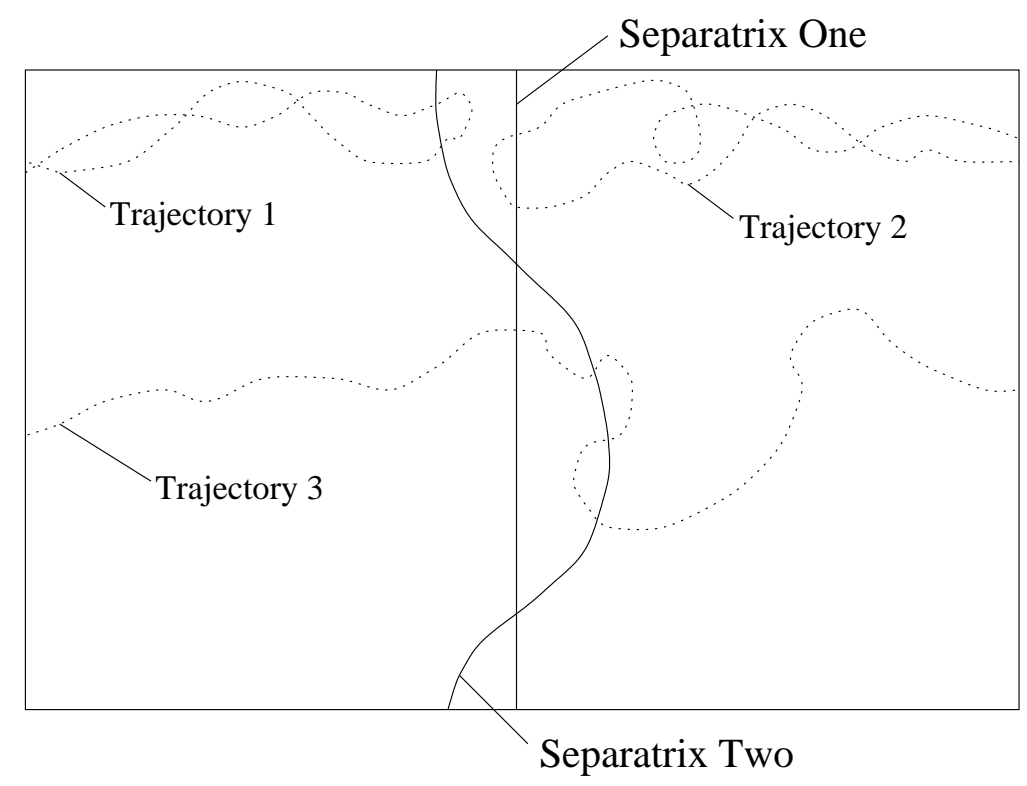

FIG. 16. cartoon of separatrices for two measurables, and some trajectories. Trajectory 1 crosses separatrix 2 but not separatrix 1; but it (therefore) crosses an even number of times, and leads to a 0 entry in determining $\mathbf{d}_{\mathcal{O} 2}$. Similarly Trajectory 2 crosses separatrix 1 but not separatrix 2 , but also does not contribute to $\mathbf{d}_{\mathcal{O} 1}$. Trajectory 3 does lead to a nucleation. It is sampled 3 times in computing $\mathbf{d}_{\mathcal{O} 2}$, each time contributing $(1 / 3)$; and sampled once in computing $\mathbf{d}_{\mathcal{O} 1}$, contributing 1. Hence it gives the same contribution to the total rate computation for each measurable.

$\mathbf{d}$, so the number of nucleations is correctly counted as 1. Again, the Boltzmann weighted amount of flux the trajectory crossing represents, is the same at each of its crossings of either separatrix. Hence if there is a larger total flux through, say, the separatrix for $\mathcal{O}_{1}$, then this individual trajectory represents a smaller fraction of that flux, and gets an appropriately smaller weight in the sampling for determining $\mathbf{d}_{\mathcal{O} 1}$. Its positive contribution to the value of $\mathbf{d}_{\mathcal{O} 1}$ is correspondingly smaller. Since the set of trajectories which mediate nucleations are the same, whichever separatrix we use to sample them, the smaller size of $\mathbf{d}$ exactly compensates for the larger flux through the separatrix. This is why the total rate computed is the same for each choice of $\mathcal{O}$.

While different observables give the same answer for the nucleation rate, they are not equally convenient as numerical tools. A good measurable must satisfy two conditions. First, it must be easy to measure it, so it can be used practically in reweighting configurations in the Monte Carlo (see subsection IVB). Second, when determining d, statistics must accumulate with a reasonable sample of configurations, which means that a reasonable fraction of trajectories through the separatrix must actually lead to bubble nucleation. Otherwise it may take an exponentially large sample of trajectories to determine $\mathbf{d}$ with good statistical accuracy. Roughly, this will require that the separatrix of the observable is close to degenerate with the separatrix of $\mathcal{O}_{\text {ideal }}$. Note that, by construction, under Langevin dynamics half of trajectories through the $\mathcal{O}_{\text {ideal }}$ separatrix lead to nucleations, and that this is the upper bound among all observables. 


\section{REFERENCES}

[1] M. Joyce, Phys. Rev. D55, 1875 (1997) hep-ph/9606223; M. Joyce and T. Prokopec, Phys. Rev. D57, 6022 (1998) [hep-ph/9709320].

[2] M. E. Shaposhnikov, Nucl. Phys. B287 757 (1987).

[3] K. Kajantie, M. Laine, K. Rummukainen and M. Shaposhnikov, Nucl. Phys. B466, 189 (1996) hep-lat/9510020.

[4] K. Farakos, K. Kajantie, K. Rummukainen and M. Shaposhnikov, Nucl. Phys. B442, 317 (1995) hep-lat/9412091.

[5] K. Kajantie, M. Laine, K. Rummukainen and M. Shaposhnikov, Nucl. Phys. B458, 90 (1996) hep-ph/9508379.

[6] G.D. Moore, Nucl. Phys. B523, 569 (1998) [hep-lat/9709053].

[7] K. Kajantie, M. Laine, K. Rummukainen and M. Shaposhnikov, Phys. Rev. Lett. 77, 2887 (1996) hep-ph/9605288].

[8] M. Gurtler, E. M. Ilgenfritz and A. Schiller, Phys. Rev. D56, 3888 (1997) heplat/9704013; ; K. Rummukainen, M. Tsypin, K. Kajantie, M. Laine and M. Shaposhnikov, Nucl. Phys. B532, 283 (1998) hep-lat/9805013;; F. Karsch, T. Neuhaus, A. Patkos and J. Rank, Nucl. Phys. B474, 217 (1996) [hep-lat/9603004].

[9] Z. Fodor, J. Hein, K. Jansen, A. Jaster and I. Montvay, Nucl. Phys. B439, 147 (1995) [hep-lat/9409017]; J. Hein and J. Heitger, Phys. Lett. B385, 242 (1996) heplat/9605009].

[10] F. Csikor, Z. Fodor and J. Heitger, Phys. Rev. Lett. 82, 21 (1999) hep-ph/9809291;

Y. Aoki, F. Csikor, Z. Fodor and A. Ukawa, Phys. Rev. D60, 013001 (1999) heplat/9901021].

[11] M. Gleiser, E. W. Kolb and R. Watkins, Nucl. Phys. B364, 411 (1991).

[12] A. E. Nelson, D. B. Kaplan and A. G. Cohen, Nucl. Phys. B373, 453 (1992).

[13] V.A. Kuzmin, V.A. Rubakov and M.E. Shaposhnikov, Phys. Lett. B155, 36 (1985).

[14] P. Arnold and L. McLerran, Phys. Rev. D 36, 581 (1987).

[15] D. Bödeker, Phys. Lett. B 426, 351 (1998) [hep-ph/9801430].

[16] G. D. Moore, Nucl. Phys. B568, 367 (2000) hep-ph/9810313.

[17] D. Bödeker, G. D. Moore and K. Rummukainen, Phys. Rev. D61, 056003 (2000) hep$\mathrm{ph} / 9907545$.

[18] P. Arnold and L. G. Yaffe, hep-ph/9912306.

[19] G. D. Moore, hep-ph/0001216.

[20] G. D. Moore, JHEP 0003, 006 (2000) [hep-ph/0001274].

[21] P. John and M. G. Schmidt, hep-ph/0002050.

[22] S. J. Huber and M. G. Schmidt, hep-ph/0003122.

[23] J. M. Cline and K. Kainulainen, hep-ph/0002272; J. M. Cline, M. Joyce and K. Kainulainen, hep-ph/0006119.

[24] A. F. Heckler, Phys. Rev. D51 405 (1995) astro-ph/9407064.

[25] D. Stauffer, A. Coniglio and D.W. Heermann, Phys. Rev. Lett. 491299 (1982); K. Binder, Phys. Rev. A, 251699 (1982); M. Acharyya and D. Stauffer, condmat/9801213|, P.A. Rikvold et al, "Dynamic Phase Transition and Hysteresis in Kinetic Ising Models," in "Computer Simulation Studies in Condensed Matter Physics XII", edited by D.P. Landau et al., Springer Proceedings in Physics Vol. 85 (Springer, Berlin, 2000). 
[26] S. Borsanyi, A. Patkos, J. Polonyi and Z. Szep, hep-th/0004059.

[27] G.D. Moore, K. Rummukainen and A. Tranberg, presented at "Strong and Electroweak Matter 2000", to be published.

[28] J. Langer, Ann. Phys. (N. Y.) 41 (1967) 108; ibid 54258 (1969).

[29] G. D. Moore, Phys. Lett. B439, 357 (1998) hep-ph/9801204.

[30] G. D. Moore, Phys. Rev. D59, 014503 (1999) hep-ph/9805264.

[31] K. Kajantie, M. Laine, K. Rummukainen and M. Shaposhnikov, Nucl. Phys. B493, 413 (1997) hep-lat/9612006.

[32] J. Ambjørn and A. Krasnitz, Phys. Lett. B 362, 97 (1995) [hep-ph/9508202].

[33] D. Bödeker, P. John, M. Laine and M. G. Schmidt, Nucl. Phys. B497 387 (1997) hep$\mathrm{ph} / 9612364$.

[34] M. Laine and K. Rummukainen, Nucl. Phys. B535 423 (1998) hep-lat/9804019; Phys. Rev. Lett. 805259 (1998) hep-ph/9804255.

[35] D. Y. Grigoriev and V. A. Rubakov, Nucl. Phys. B299 67 (1988).

[36] D. Bödeker, L. McLerran and A. Smilga, Phys. Rev. D52, 4675 (1995) [hep-th/9504123].

[37] P. Arnold, D. Son and L.G. Yaffe, Phys. Rev. D55, 6264 (1997) [hep-ph/9609481].

[38] E. Braaten and R. Pisarski, Nucl. Phys. B337, 569 (1990); J. Frenkel and J. Taylor, Nucl. Phys. B334, 199 (1990); J. Taylor and S. Wong, Nucl. Phys. B346, 115 (1990); J. Frenkel and J. Taylor, Nucl. Phys. B 374, 156 (1992); E. Braaten and R. Pisarski, Phys. Rev. D 45, 1827 (1992); J.P. Blaizot and E. Iancu, Phys. Rev. Lett. 70, 3376 (1993) [hep-ph/9301236]; Nucl. Phys. B417, 608 (1994) hep-ph/9306294; V.P. Nair, Phys. Rev. D48, 3432 (1993) [hep-ph/9307326].

[39] G. D. Moore, C. Hu and B. Müller, Phys. Rev. D58, 045001 (1998) hep-ph/9710436.

[40] C. R. Hu and B. Müller, Phys. Lett. B409, 377 (1997) hep-ph/9611292.

[41] E. Iancu, hep-ph/9710543.

[42] D. Bödeker, hep-ph/9905239; hep-ph/9903478.

[43] P. Arnold, D. T. Son and L. G. Yaffe, Phys. Rev. D59, 105020 (1999); Phys. Rev. D60, 025007 (1999) hep-ph/9901304.

[44] D. F. Litim and C. Manuel, Phys. Rev. Lett. 82, 4981 (1999) hep-ph/9902430; Nucl. Phys. B562, 237 (1999) hep-ph/9906210; Phys. Rev. D61, 125004 (2000) hepph/9910348.

[45] K. Binder, Phys. Rev. A 251699 (1982).

[46] M. Gurtler, E. M. Ilgenfritz, J. Kripfganz, H. Perlt and A. Schiller, Nucl. Phys. B483, 383 (1997) |hep-lat/9605042]; M. Gurtler, E. M. Ilgenfritz and A. Schiller, Eur. Phys. J. C1, 363 (1998) hep-lat/9702020.

[47] B. Bunk, Int. J. Mod. Phys. C 3889 (1992).

[48] Y. Iwasaki, K. Kanaya, L. Kärkkäinen, K. Rummukainen and T. Yoshié, Phys. Rev. D 493540 (1994).

[49] G. D. Moore and N. Turok, Phys. Rev. D55 6538 (1997) hep-ph/9608350.

[50] K. Enqvist, J. Ignatius, K. Kajantie and K. Rummukainen, Phys. Rev. D45, 3415 (1992).

[51] N. Turok, Phys. Rev. Lett. 681803 (1992).

[52] M. Dine, R. G. Leigh, P. Huet, A. Linde and D. Linde, Phys. Rev. D46, 550 (1992) hep-ph/9203203.

[53] A. Strumia and N. Tetradis, Nucl. Phys. B554, 697 (1999) hep-ph/9811438. 
[54] G. Munster and S. Rotsch, Eur. Phys. J. C12, 161 (2000) cond-mat/9908246.

[55] J. Berges, N. Tetradis and C. Wetterich, Phys. Lett. B393, 387 (1997) hep-ph/9610354.

[56] G. Munster, A. Strumia and N. Tetradis, Phys. Lett. A271, 80 (2000) condmat/0002278.

[57] Z. Fodor and A. Hebecker, Nucl. Phys. B432, 127 (1994) hep-ph/9403219.

[58] K. Farakos, K. Kajantie, K. Rummukainen and M. Shaposhnikov, Nucl. Phys. B425, 67 (1994) hep-ph/9404201.

[59] W. Buchmuller, Z. Fodor and A. Hebecker, Phys. Lett. B331, 131 (1994) hepph/9403391].

[60] P. Arnold and O. Espinosa, Phys. Rev. D47, 3546 (1993) hep-ph/9212235.

[61] D. Bödeker, W. Buchmuller, Z. Fodor and T. Helbig, Nucl. Phys. B423, 171 (1994) hep-ph/9311346.

[62] J. Kripfganz, A. Laser and M. G. Schmidt, Z. Phys. C73 353 (1997) hep-ph/9512340.

[63] J. Baacke, Phys. Rev. D52, 6760 (1995) [hep-ph/9503350].

[64] A. Parnachev and L. G. Yaffe, hep-th/0005269.

[65] P. Arnold, D. T. Son and L. G. Yaffe, Phys. Rev. D60, 025007 (1999) hep-ph/9901304.

[66] P. Arnold, Phys. Rev. E61, 6091 (2000) [hep-ph/9912208.

[67] S. Y. Khlebnikov and M. E. Shaposhnikov, Nucl. Phys. B308 885 (1988). 